\title{
A TYPE OF FULL MULTIGRID METHOD FOR NON-SELFADJOINT STEKLOV EIGENVALUE PROBLEMS IN INVERSE SCATTERING
}

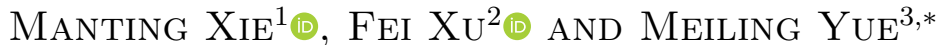

\begin{abstract}
In this paper, a type of full multigrid method is proposed to solve non-selfadjoint Steklov eigenvalue problems. Multigrid iterations for corresponding selfadjoint and positive definite boundary value problems generate proper iterate solutions that are subsequently added to the coarsest finite element space in order to improve approximate eigenpairs on the current mesh. Based on this full multigrid, we propose a new type of adaptive finite element method for non-selfadjoint Steklov eigenvalue problems. We prove that the computational work of these new schemes are almost optimal, the same as solving the corresponding positive definite selfadjoint boundary value problems. In this case, these type of iteration schemes certainly improve the overfull efficiency of solving the non-selfadjoint Steklov eigenvalue problem. Some numerical examples are provided to validate the theoretical results and the efficiency of this proposed scheme.
\end{abstract}

Mathematics Subject Classification. 35Q99, 65N30, 65M12, 65M70.

Received August 19, 2020. Accepted July 28, 2021.

\section{INTRODUCTION}

Inverse scattering problems for inhomogeneous media have many applications, such as medical imaging and nondestructive testing and so on. Recently, non-selfadjoint Steklov eigenvalues have been widely applied in the inverse scattering problem. Particularly, it can be used to reconstruct the shape of the obstacle and estimate the index of refraction of the inhomogeneous medium [9]. Compared with the transmission eigenvalue problem, non-selfadjoint Steklov eigenvalues associated with the scattering problem have many advantages [30], and the potential to work for a wider class of problems, such as the surface waves, mechanical oscillators immersed in a viscous fluid and the vibration modes of a structure in contact with an incompressible fluid $[10,11,27,31]$.

\footnotetext{
Keywords and phrases. Non-selfadjoint steklov eigenvalue problem, full multigrid method, multilevel correction method, adaptive finite element method.

1 Center for Applied Mathematics, Tianjin University, Tianjin 300072, China. mtxie@tju.edu.cn

2 Beijing Institute for Scientiic and Engineering Computing, College of applied sciences, Beijing University of Technology, Beijing 100124, China.

3 School of Mathematics and Statistics, Beijing Technology and Business University, Beijing 100048, China.

${ }^{*}$ Corresponding author: yuemeiling@lsec.cc.ac.cn
} 
As we know, there are many efficient methods for solving selfadjoint Steklov eigenvalue problems $[1,4,21$, $34,37,49]$. However, the non-selfadjoint Steklov eigenvalue problem for inverse scattering will lead to a nonHermitian matrices, the multiplicities are not known, and the computation of complex eigenvalues and eigenvectors of such non-Hermitian matrices are quite challenging. The differential operator corresponding to this problem is non-selfadjoint and the associated weak formulation does not satisfy $H^{1}$-elliptic condition, which are the main differences from those studied before. Hence, extensions of the methods for selfadjoint eigenvalue problems to the non-selfadjoint ones are not trivial [48]. [9] studies the mathematical properties of non-selfadjoint Steklov eigenvalue problems and its conforming finite element approximation, later [31] an explicit convergence estimate for approximate eigenvalues. A two-grid method for this problem have been proposed in [5]. In [46], the authors use the complementary technique $[40,41]$ to give a new type of adaptive method. [32] gives a discontinuous Galerkin methods for non-selfadjoint Steklov eigenvalue problem. In this paper, we aim to construct a type of full multigrid method for non-selfadjoint Steklov eigenvalue problems.

In recent ten years, the multilevel correction method for eigenvalue problems has been proposed in $[29,36]$ and applied in many useful eigenvalue problems, such as nonlinear eigenvalue problems [25, 26], biharmonic eigenvalue problem [50], nonsymmetric eigenvalue problem [42,48], Fredholm integral eigenvalue problems [43], Bose-Einstein Condensates [39], Kohn-Sham equation [24], interior transmission eigenvalue problem [38] and so on. Recently, [44] propose a parallel multilevel correction method for linear selfadjoint eigenvalue problems. Especially, multilevel correction method has been applied to non-selfadjoint Steklov eigenvalue problems in [51]. As we know, the multigrid method $[3,7,8,20]$ as an efficient preconditioners provide an optimal order algorithm for solving boundary value problems. Hence, the aim of this paper is to present a full multigrid method $[15,26,44]$ (sometimes also referred to as nested finite element method) for solving non-selfadjoint Steklov eigenvalue problems based on the combination of the multilevel correction method and the multigrid iteration for boundary value problems. Comparing with the method in $[29,36,37,51]$, we do not need to solve the linear boundary value problem exactly in each correction step in this paper. Some multigrid iteration steps are used to get an approximate solution. In this new version of multigrid method, solving non-selfadjoint Steklov eigenvalue problems will not be much more difficult than the multigrid scheme for the corresponding positive definite selfadjoint boundary value problems.

The adaptive finite element method (AFEM) has been widely used to solve singular partial differential equations, which can generate a sequence of optimal triangulations by refining those elements where the errors, as the local error estimators indicate, are relatively large. The AFEM is really an effective way to make efficient use of given computational resources. In addition to being widely used in boundary value problems $[13,14,33,35]$, AFEM is also a very useful and efficient way for solving large-scale eigenvalue problems $[12,18,19,22]$. It should be noted that the optimal complexity of AFEM means the discretization scale is optimal but not that the computational work is optimal, which is the motivation of this paper. Recently, [23,24] propose an efficient AFEM based on multilevel correction method. From this idea, we give a new type of AFEM based on full multigrid. In this method, solving non-selfadjoint Steklov eigenvalue problem only includes solving the associated positive definite selfadjoint boundary value problems on a series of adaptively refined partitions by multigrid method and the non-selfadjoint Steklov eigenvalue problem with a coarse mesh. Comparing with the standard multigrid method, we only need to do smoothing steps on the newly refined elements and their neighbors. The dimension of the coarse mesh for determining approximate eigenpairs will remain unchanged during the adaptive process, thus this computational time can be ignored as the size of mesh becomes smaller after some refinement steps. Hence, the main computation will be spent on the positive definite selfadjoint boundary value problems on adaptive spaces, and the cost of this new AFEM can be improved to be almost optimal.

An outline of the paper goes as follows. In Section 2, we introduce the finite element method for nonselfadjoint Steklov eigenvalue problems. A type of full multigrid method based on the multilevel correction scheme is presented and analyzed in Section 3. Section 4 is devoted to giving a new type of AFEM based on full multigrid proposed in Section 3. In Section 5, three numerical examples are presented to validate the efficiency of the proposed method. Finally, some concluding remarks are given in the last section. 


\section{Discretization By Finite Element Method}

In this section, we introduce the concerned nonsymmetric eigenvalue problem and its corresponding finite element method. The standard notation for the Sobolev spaces $W^{s, p}(\Omega)$ and their associated norms $\|\cdot\|_{s, p, \Omega}$ and seminorms $|\cdot|_{s, p, \Omega}$ will be used (see, e.g. $\left.[8,16]\right)$. For $p=2$, we denote $H^{s}(\Omega)=W^{s, 2}(\Omega),\|\cdot\|_{s, \Omega}=\|\cdot\|_{s, 2, \Omega}$ for simplicity. In this paper, $\|\cdot\|_{s, \Omega}$ are abbreviated to $\|\cdot\|_{s}$, and the letter $C$ (with or without subscripts) denotes a generic positive constant which may be different at its different occurrences through the paper. For convenience, the symbols $\lesssim, \gtrsim$ and $\approx$ will be used in this paper. These $x_{1} \lesssim y_{1}, x_{2} \gtrsim y_{2}$ and $x_{3} \approx y_{3}$, mean that $x_{1} \leq \widetilde{C}_{1} y_{1}, x_{2} \geq \widetilde{c}_{2} y_{2}$ and $\widetilde{c}_{3} x_{3} \leq y_{3} \leq \widetilde{C}_{3} x_{3}$ for some constants $\widetilde{C}_{1}, \widetilde{c}_{2}, \widetilde{c}_{3}$ and $\widetilde{C}_{3}$ that are independent of mesh size.

\subsection{Non-selfadjoint Steklov eigenvalue problems}

In this paper, we consider the following non-selfadjoint Steklov eigenvalue problem to find $\lambda \in \mathbb{C}$ and $u \in H^{1}(\Omega)$ such that

$$
\left\{\begin{aligned}
\nabla \cdot(\mathcal{A} \nabla u)+\kappa^{2} n(x) u & =0, & & \text { in } \Omega, \\
\frac{\partial u}{\partial \nu}+\lambda u & =0, & & \text { on } \partial \Omega,
\end{aligned}\right.
$$

where $\Omega \subset \mathbb{R}^{2}$ is a bounded polygonal domain with Lipshitz boundary $\partial \Omega$ and $\nu$ be the unit outward normal to $\partial \Omega, \mathcal{A}$ is a uniformly bounded symmetric positive definite matrix function defined on $\Omega, \kappa$ is the wavenumber and $n(x)$ is the index of refraction. Assume that $n=n(x)$ is a bounded complex valued function given by

$$
n(x)=n_{1}(x)+\mathrm{i} \frac{n_{2}(x)}{\kappa},
$$

where $\mathrm{i}=\sqrt{-1}, n_{1}(x)>0$ and $n_{2}(x) \geq 0$ are bounded smooth functions. Set $V=H^{1}(\Omega)$.

For the aim of finite element discretization, we define the corresponding weak form of (2.1) as follows: Find $(\lambda, u) \in \mathbb{C} \times V, u \neq 0$, such that

$$
a(u, v)=-\lambda b(u, v), \quad \forall v \in V
$$

where

$$
\begin{aligned}
& a(u, v)=(\mathcal{A} \nabla u, \nabla v)-\kappa^{2}(n u, v), \\
& b(u, v)=\int_{\partial \Omega} u \bar{v} \mathrm{~d} s,
\end{aligned}
$$

being two continuous sesquilinear forms, with

$$
(\phi, \psi)=\int_{\Omega} \phi \bar{\psi} \mathrm{d} x
$$

and overline denoting the complex conjugate of a function.

For any $g \in H^{1}(\Omega), b(f, g)$ has a continuous extension to $f \in H^{-1 / 2}(\partial \Omega)$ so that $b(f, g)$ is continuous on $H^{-1 / 2}(\partial \Omega) \times H^{1 / 2}(\partial \Omega)$. For convenience, we define a $H^{1}(\Omega)$ inner product as follows

$$
a_{s}(w, v):=(\mathcal{A} \nabla w, \nabla v)+(w, v), \quad \forall w, v \in V,
$$

and the following ellipticity holds

$$
\frac{1}{C_{a}^{2}}\|v\|_{1}^{2} \leq a_{s}(v, v), \quad \forall v \in V
$$

For the non-selfadjoint Steklov eigenvalue problem (2.2), there exists the corresponding adjoint eigenvalue problem $(c f .[31])$ : Find $\left(\lambda^{*}, u^{*}\right) \in \mathbb{C} \times V$ such that

$$
a\left(v, u^{*}\right)=-b\left(v, \lambda^{*} u^{*}\right)=-\overline{\lambda^{*}} b\left(v, u^{*}\right), \quad \forall v \in V .
$$


Note that the (2.2) and (2.4) are also connected via $\lambda=\overline{\lambda^{*}}$.

For simplicity, we only consider the nondefective eigenvalues (the ascent equals to 1 ) of the non-adjoint Steklov eigenvalue problem. Thus, the algebraic multiplicity equals to the geometric multiplicity and the generalized eigenspace is the same as the eigenspace. More details about the nonsymmetric eigenvalue problems, please refer to $[2,41,42,46,48]$.

\subsection{Finite element method}

Now, we introduce the finite element method ( $c f .[2])$ for the non-selfadjoint Steklov eigenvalue problem (2.2) and its corresponding adjoint problem (2.4).

First, we decompose the computing domain $\Omega \subset \mathbb{R}^{d}(d=2,3)$ into shape-regular triangles or rectangles for $d=2$ (tetrahedrons or hexahedrons for $d=3$ ) and the diameter of a cell $K \in \mathcal{T}_{h}$ is denoted by $h_{K}$. The mesh diameter $h$ describes the maximum diameter of all cells $K \in \mathcal{T}_{h}$. Based on the mesh $\mathcal{T}_{h}$, we construct the conforming finite element space denoted by $V_{h} \subset V$. For simplicity, we only consider the linear Lagrange conforming finite element space which is defined as follows

$$
V_{h}=\left\{v_{h} \in C(\bar{\Omega})\left|v_{h}\right|_{K} \in \mathcal{P}_{1}(K), \quad \forall K \in \mathcal{T}_{h}\right\},
$$

where $\mathcal{P}_{1}(K)$ denotes the space of polynomials of degree $\leq 1$.

The standard finite element method for $(2.2)$ is to solve the following eigenvalue problem: Find $\left(\widehat{\lambda}_{h}, \widehat{u}_{h}\right) \in$ $\mathbb{C} \times V_{h}$ such that

$$
a\left(\widehat{u}_{h}, v_{h}\right)=-\widehat{\lambda}_{h} b\left(\widehat{u}_{h}, v_{h}\right), \quad \forall v_{h} \in V_{h} .
$$

We give the discretization of the adjoint problem (2.4) in the same finite element space: Find $\left(\widehat{\lambda}_{h}^{*}, \widehat{u}_{h}^{*}\right) \in \mathbb{C} \times V_{h}$ such that

$$
a\left(v_{h}, \widehat{u}_{h}^{*}\right)=-\overline{\widehat{\lambda}_{h}^{*}} b\left(v_{h}, \widehat{u}_{h}^{*}\right), \quad \forall v_{h} \in V_{h},
$$

also we have the relation $\widehat{\lambda}_{h}=\overline{\widehat{\lambda}_{h}^{*}}$. Hereafter, we use the triple $\left(\widehat{\lambda}_{h}, \widehat{u}_{h}, \widehat{u}_{h}^{*}\right)$ to denote the finite element method approximate eigenpair of the non-selfadjoint Steklov eigenvalue problems (2.2) and (2.4).

Define $\eta_{a}\left(V_{h}\right)$ and $\eta_{a}^{*}\left(V_{h}\right)$ as

$$
\begin{aligned}
\eta_{a}\left(V_{h}\right):= & \sup _{\substack{f \in H^{1 / 2}(\partial \Omega) \\
\|f\|_{1 / 2, \partial \Omega}=1}} \inf _{v_{h} \in V_{h}}\left\|T f-v_{h}\right\|_{1}, \\
\eta_{a}^{*}\left(V_{h}\right):= & \sup _{\substack{f \in H^{1 / 2}(\partial \Omega) \\
\|f\|_{1 / 2, \partial \Omega}=1}} \inf _{v_{h} \in V_{h}}\left\|T_{*} f-v_{h}\right\|_{1},
\end{aligned}
$$

where the operators $T$ and $T_{*}: H^{-1 / 2}(\partial \Omega) \rightarrow V$ are defined by

$$
\begin{aligned}
a(T f, v) & =b(f, v), \quad \forall f \in H^{-1 / 2}(\partial \Omega) \text { and } \forall v \in V, \\
a\left(v, T_{*} f\right) & =b(f, v), \quad \forall f \in H^{-1 / 2}(\partial \Omega) \text { and } \forall v \in V .
\end{aligned}
$$

Introduce the following Neumann eigenvalue problem:

$$
\left\{\begin{aligned}
\nabla \cdot(\mathcal{A} \nabla u)+\kappa^{2} n(x) u & =0, & & \text { in } \Omega \\
\frac{\partial u}{\partial \nu} & =0, & & \text { on } \partial \Omega .
\end{aligned}\right.
$$

When $\kappa^{2}$ is not a Neumann eigenvalue of the above eigenvalue problem, we have that for any $f \in H^{-1 / 2}(\partial \Omega)$, there exist unique solution for (2.8) and (2.9).

In order to give the convergence order of eigenpair approximations by the finite element method, we need the following regularity result for the boundary value problem (2.8). 
Lemma $2.1([5,6])$. For the Steklov-type boundary value problem (2.8), if $f \in L^{2}(\partial \Omega)$, then $T f \in H^{1+\sigma / 2}(\Omega)$ and

$$
\|T f\|_{1+\sigma / 2} \leq C\|f\|_{0, \partial \Omega} .
$$

Furthermore, if $f \in H^{1 / 2}(\partial \Omega)$, we have $T f \in H^{1+\sigma}(\Omega)$ and

$$
\|T f\|_{1+\sigma} \leq C\|f\|_{1 / 2, \partial \Omega} .
$$

Here $\sigma=1$ if $\Omega$ is convex and $\sigma<\pi / \omega$ (with $\omega$ being the largest inner angle of $\partial \Omega$ ) (see, e.g., Grisvard, 1986).

Let $M(\lambda)$ and $M^{*}(\lambda)$ denote two eigenspaces corresponding to the eigenvalue $\lambda$ of (2.2) and (2.4), respectively,

$$
\begin{aligned}
M(\lambda) & =\{u \in V: u \text { is an eigenfunction of (2.2) corresponding to } \lambda\}, \\
M^{*}(\lambda) & =\left\{u^{*} \in V: u^{*} \text { is an eigenfunction of (2.4) corresponding to } \lambda\right\} .
\end{aligned}
$$

Then, we introduce the following notation for error estimation

$$
\begin{aligned}
\delta\left(u, V_{h}\right) & :=\sup _{u \in M(\lambda),\|u\|_{0}=1} \inf _{v_{h} \in V_{h}}\left\|u-v_{h}\right\|_{1}, \\
\delta\left(u^{*}, V_{h}\right) & :=\sup _{u^{*} \in M^{*}(\lambda),\left\|u^{*}\right\|_{0}=1} \inf _{v_{h} \in V_{h}}\left\|u^{*}-v_{h}\right\|_{1} .
\end{aligned}
$$

Since the ascent of the non-selfadjoint Steklov eigenvalue problem equals to 1, we have the following error estimates.

Lemma $2.2([5,31])$.

(i) For any eigenfunction approximations $\widehat{u}_{h}$ and $\widehat{u}_{h}^{*}$ of (2.6) and (2.7), respectively, there exist eigenfunctions $u$ and $u^{*}$ of (2.2) and (2.4), such that

$$
\begin{aligned}
\left\|u-\widehat{u}_{h}\right\|_{1} & \leq\left(1+C_{\lambda}\left(\eta_{a}\left(V_{h}\right)+\delta\left(u^{*}, V_{h}\right)\right)\right) \delta\left(u, V_{h}\right), \\
\left\|u^{*}-\widehat{u}_{h}^{*}\right\|_{1} & \leq\left(1+C_{\lambda}\left(\eta_{a}^{*}\left(V_{h}\right)+\delta\left(u, V_{h}\right)\right)\right) \delta\left(u^{*}, V_{h}\right),
\end{aligned}
$$

Furthermore,

$$
\begin{aligned}
\left\|u-\widehat{u}_{h}\right\|_{0} & \leq C_{\lambda} \eta_{a}\left(V_{h}\right) \delta\left(u, V_{h}\right), \\
\left\|u^{*}-\widehat{u}_{h}^{*}\right\|_{0} & \leq C_{\lambda} \eta_{a}^{*}\left(V_{h}\right) \delta\left(u^{*}, V_{h}\right),
\end{aligned}
$$

and

$$
\begin{aligned}
\left\|u-\widehat{u}_{h}\right\|_{-1 / 2, \partial \Omega} & \leq C_{\lambda} \eta_{a}\left(V_{h}\right) \delta\left(u, V_{h}\right), \\
\left\|u^{*}-\widehat{u}_{h}^{*}\right\|_{-1 / 2, \partial \Omega} & \leq C_{\lambda} \eta_{a}^{*}\left(V_{h}\right) \delta\left(u^{*}, V_{h}\right) .
\end{aligned}
$$

(ii) For each eigenvalue, we have

$$
\left|\lambda-\widehat{\lambda}_{h}\right| \leq C_{\lambda} \delta\left(u, V_{h}\right) \delta\left(u^{*}, V_{h}\right) .
$$

Here and hereafter $C_{\lambda}$ is some constant depending on eigenvalue $\lambda$ but independent of the mesh size $h$.

Corollary 2.3. Based on the regularity (2.10), if $V_{h}$ is the linear finite element space, then we have the following estimates for $\eta_{a}\left(V_{h}\right), \eta_{a}^{*}\left(V_{h}\right), \delta\left(u, V_{h}\right)$ and $\delta\left(u^{*}, V_{h}\right)$ :

$$
\begin{aligned}
\eta_{a}\left(V_{h}\right) & \leq C_{\sigma} h^{\sigma}, \\
\eta_{a}^{*}\left(V_{h}\right) & \leq C_{\sigma} h^{\sigma}, \\
\delta\left(u, V_{h}\right) & \leq C_{\sigma} h^{\sigma}, \\
\delta\left(u^{*}, V_{h}\right) & \leq C_{\sigma} h^{\sigma},
\end{aligned}
$$

$C_{\sigma}$ is some constant depending on $\left(\lambda, u, u^{*}\right)$ but independent of the mesh size $h$. 
Lemma 2.4 ([47] Lem. 4.1). Suppose that $\left(\widehat{\lambda}_{h}, \widehat{u}_{h}\right) \in \mathbb{C} \times V$ is an eigenpair of (2.6). Let $u_{h}^{\perp}$ be the orthogonal projection of $\widehat{u}_{h}$ to $M_{h}^{*}(\lambda)$ in the sense of inner product $b(\cdot, \cdot)$, and let

$$
\widehat{u}_{h}^{*}=\frac{u_{h}^{\perp}}{\left\|u \frac{\perp}{h}\right\|_{0, \partial \Omega}},
$$

and $\widehat{\lambda}_{h}^{*}=\bar{\lambda}_{h}$. Then when $h$ is small enough $\left|b\left(\widehat{u}_{h}, \widehat{u}_{h}^{*}\right)\right| \geq C_{0} /\left|\widehat{\lambda}_{h}\right|$.

Remark 2.5. We can use the algorithm in Remark 4.1 in [47] to compute $u_{h}^{\perp}$ and then obtain $\widehat{u}_{h}^{*}$.

Lemma $2.6([46,51])$. Assume $(\lambda, u) \in \mathbb{C} \times V$ and $\left(\lambda, u^{*}\right) \in \mathbb{C} \times V$ satisfy (2.2) and (2.4), respectively, and suppose $w, w^{*} \in V$ such that $b\left(w, w^{*}\right) \neq 0$. Let us define

$$
\underline{\lambda}=\frac{a\left(w, w^{*}\right)}{b\left(w, w^{*}\right)}
$$

Then we have following expansion

$$
\underline{\lambda}-\lambda=\frac{-a\left(w-u, w^{*}-u^{*}\right)-\lambda b\left(w-u, w^{*}-u^{*}\right)}{b\left(w, w^{*}\right)} .
$$

\section{Full multigrid Algorithm for nOn-Selfadjoint Steklov eigenvalue Problem}

In this section, a type of full multigrid method is presented. In order to describe the full multigrid method, we first introduce the sequence of finite element spaces. We generate a coarse mesh $\mathcal{T}_{H}$ with the mesh size $H$ and the coarse linear finite element space $V_{H}$ is defined on the mesh $\mathcal{T}_{H}$. Then a sequence of triangulations $\mathcal{T}_{h_{k}}$ of $\Omega \subset \mathbb{R}^{d}$ is determined as follows. Suppose $\mathcal{T}_{h_{1}}$ (produced from $\mathcal{T}_{H}$ by regular refinements) is given and let $\mathcal{T}_{h_{k}}$ be obtained from $\mathcal{T}_{h_{k-1}}$ via $\zeta$ times regular refinements (produce $\left(\beta^{d}\right)^{\zeta}$ subelements) such that

$$
h_{k}=\left(\frac{1}{\beta}\right)^{\zeta} h_{k-1}, \quad k=2, \cdots, n,
$$

where the positive number $\beta$ denotes the refinement index and larger than 1 (usually for classical bisection refinement $\beta=2$ and $\zeta=1$ ). Based on this sequence of meshes, the corresponding nested linear finite element spaces can be built such that

$$
V_{H} \subseteq V_{h_{1}} \subset V_{h_{2}} \subset \cdots \subset V_{h_{n}} .
$$

The sequence of finite element spaces $V_{h_{1}} \subset V_{h_{2}} \subset \cdots \subset V_{h_{n}}$ and the finite element space $V_{H}$ have the following relations of approximation accuracy $(c f .[8,16])$ : for $k=2, \cdots, n$

$$
\begin{aligned}
\frac{1}{C_{\delta}}\left(\frac{1}{\beta}\right)^{\zeta} \delta\left(u, V_{h_{k-1}}\right) & \leq \delta\left(u, V_{h_{k}}\right) \leq \tilde{C}_{\delta}\left(\frac{1}{\beta}\right)^{\zeta} \delta\left(u, V_{h_{k-1}}\right), \\
\frac{1}{C_{\delta}^{*}}\left(\frac{1}{\beta}\right)^{\zeta} \delta\left(u^{*}, V_{h_{k-1}}\right) \leq \delta\left(u^{*}, V_{h_{k}}\right) & \leq \tilde{C}_{\delta}^{*}\left(\frac{1}{\beta}\right)^{\zeta} \delta\left(u^{*}, V_{h_{k-1}}\right) .
\end{aligned}
$$




\subsection{One correction step}

First, we present the one correction step to improve the accuracy of the given eigenvalue and eigenfunction approximation. This correction contains solving an auxiliary boundary value problem inexactly on the current finite element space and an eigenvalue problem on a slight extension of the coarsest finite element space. In this paper, we use $\left(\widehat{\lambda}_{h}, \widehat{u}_{h}, \widehat{u}_{h}^{*}\right)$ to denote the solution of direct finite element method, see Lemma 2.2 .

Assume that we have obtained the algebraic eigenpair approximations

$$
\left(\lambda_{h_{k}}^{(\ell)}, u_{h_{k}}^{(\ell)}, u_{h_{k}}^{*(\ell)}\right) \in \mathbb{C} \times V_{h_{k}} \times V_{h_{k}}
$$

where $(\ell)$ denotes the $\ell$-th iteration step in the $k$-th level finite element space $V_{h_{k}}$. In this subsection, a type of correction step to improve the accuracy of the current eigenpair approximation $\left(\lambda_{h_{k}}^{(\ell)}, u_{h_{k}}^{(\ell)}, u_{h_{k}}^{*(\ell)}\right)$ will be given as follows.

\section{Algorithm 3.1. One Correction Step}

1. Define the following auxiliary boundary value problems:

Find $\check{u}_{h_{k}}^{(\ell+1)} \in V_{h_{k}}$ such that

$$
a_{s}\left(\check{u}_{h_{k}}^{(\ell+1)}, v_{h_{k}}\right)=-\lambda_{h_{k}}^{(\ell)} b\left(u_{h_{k}}^{(\ell)}, v_{h_{k}}\right)+\left(\left(1+\kappa^{2} n(x)\right) u_{h_{k}}^{(\ell)}, v_{h_{k}}\right), \quad \forall v_{h_{k}} \in V_{h_{k}} .
$$

Find $\check{u}_{h_{k}}^{*(\ell+1)} \in V_{h_{k}}$ such that

$$
a_{s}\left(v_{h_{k}}, \check{u}_{h_{k}}^{*(\ell+1)}\right)=-\lambda_{h_{k}}^{(\ell)} b\left(v_{h_{k}}, u_{h_{k}}^{*(\ell)}\right)+\left(v_{h_{k}},\left(1+\kappa^{2} \bar{n}(x)\right) u_{h_{k}}^{*(\ell)}\right), \quad \forall v_{h_{k}} \in V_{h_{k}} .
$$

Solve (3.5) and (3.6) by performing $m$ multigrid iteration steps with the initial guess value $u_{h_{k}}^{(\ell)}$ and $u_{h_{k}}^{*(\ell)}$ to obtain two new approximate solutions $\widetilde{u}_{h_{k}}^{(\ell+1)}$ and $\widetilde{u}_{h_{k}}^{*(\ell+1)}$, respectively.

2. Define $V_{H, h_{k}}=V_{H}+\operatorname{span}\left\{\widetilde{u}_{h_{k}}^{(\ell+1)}\right\}$ and $V_{H, h_{k}}^{*}=V_{H}+\operatorname{span}\left\{\widetilde{u}_{h_{k}}^{*(\ell+1)}\right\}$, and solve the following eigenvalue problem:

Find $\left(\lambda_{h_{k}}^{(\ell+1)}, u_{h_{k}}^{(\ell+1)}\right) \in \mathbb{C} \times V_{H, h_{k}}$ such that

$$
a\left(u_{h_{k}}^{(\ell+1)}, v_{H, h_{k}}\right)=-\lambda_{h_{k}}^{(\ell+1)} b\left(u_{h_{k}}^{(\ell+1)}, v_{H, h_{k}}\right), \quad \forall v_{H, h_{k}} \in V_{H, h_{k}} .
$$

Find $u_{h_{k}}^{*(\ell+1)} \in V_{H, h_{k}}^{*}$ according to Lemma 2.4 and Remark 2.5.

In order to simplify the notation and summarize the above two steps, we define

$$
\left(\lambda_{h_{k}}^{(\ell+1)}, u_{h_{k}}^{(\ell+1)}, u_{h_{k}}^{*(\ell+1)}\right)=\operatorname{EigenMG}\left(V_{H}, \lambda_{h_{k}}^{(\ell)}, u_{h_{k}}^{(\ell)}, u_{h_{k}}^{*(\ell)}, V_{h_{k}}, m\right) .
$$

Remark 3.1. Here we first use finite element method to discretize (3.7) and then use implicitly restarted Arnoldi methods (e.g., ARPACK or "eigs/sptarn" function in MATLAB) to solve the algebraic eigenvalue problem.

Lemma 3.2 ([3, 7, 8, 20]). Performing $m$ multigrid iteration to solve linear Equations (3.5) and (3.6) with the initial guess value $u_{h_{k}}^{(\ell)}$ and $u_{h_{k}}^{*(\ell)}$, we obtain two new approximate solutions $\widetilde{u}_{h_{k}}^{(\ell+1)}$ and $\widetilde{u}_{h_{k}}^{*(\ell+1)}$ which have the following uniform contraction rate:

$$
\begin{aligned}
&\left\|\check{u}_{h_{k}}^{(\ell+1)}-\widetilde{u}_{h_{k}}^{(\ell+1)}\right\|_{1} \leq \theta\left\|\check{u}_{h_{k}}^{(\ell+1)}-u_{h_{k}}^{(\ell)}\right\|_{1}, \\
&\left\|\check{u}_{h_{k}}^{*(\ell+1)}-\widetilde{u}_{h_{k}}^{*(\ell+1)}\right\|_{1} \leq \theta\left\|\check{u}_{h_{k}}^{*(\ell+1)}-u_{h_{k}}^{*(\ell)}\right\|_{1},
\end{aligned}
$$


where $\theta<1$ is a fixed constant independent of the mesh size $h_{k}$ and iteration step $\ell$.

Now, we turn to give the error estimates of Algorithm 3.1, which indicates that the accuracy of numerical eigenpair can be improved after one correction step.

Theorem 3.3. Assume the given eigenpair approximation $\left(\lambda_{h_{k}}^{(\ell)}, u_{h_{k}}^{(\ell)}, u_{h_{k}}^{*(\ell)}\right)$ has following estimates

$$
\begin{aligned}
\left\|\widehat{u}_{h_{k}}-u_{h_{k}}^{(\ell)}\right\|_{0} & \leq \check{C} \eta_{a}\left(V_{H}\right)\left\|\widehat{u}_{h_{k}}-u_{h_{k}}^{(\ell)}\right\|_{1}, \\
\left\|\widehat{u}_{h_{k}}^{*}-u_{h_{k}}^{*(\ell)}\right\|_{0} & \leq \check{C} \eta_{a}^{*}\left(V_{H}\right)\left\|\widehat{u}_{h_{k}}^{*}-u_{h_{k}}^{*(\ell)}\right\|_{1}, \\
\left\|\widehat{u}_{h_{k}}-u_{h_{k}}^{(\ell)}\right\|_{-1 / 2, \partial \Omega} & \leq \check{C} \eta_{a}\left(V_{H}\right)\left\|\widehat{u}_{h_{k}}-u_{h_{k}}^{(\ell)}\right\|_{1}, \\
\left\|\widehat{u}_{h_{k}}^{*}-u_{h_{k}}^{*(\ell)}\right\|_{-1 / 2, \partial \Omega} & \leq \check{C} \eta_{a}^{*}\left(V_{H}\right)\left\|\widehat{u}_{h_{k}}^{*}-u_{h_{k}}^{*(\ell)}\right\|_{1}, \\
\left|\widehat{\lambda}_{h_{k}}-\lambda_{h_{k}}^{(\ell)}\right| & \leq \check{C}\left\|\widehat{u}_{h_{k}}-u_{h_{k}}^{(\ell)}\right\|_{1}\left\|\widehat{u}_{h_{k}}^{*}-u_{h_{k}}^{*(\ell)}\right\|_{1} .
\end{aligned}
$$

After the One Correction Step defined in Algorithm 3.1, the resultant approximate eigenpair $\left(\lambda_{h_{k}}^{(\ell+1)}, u_{h_{k}}^{(\ell+1)}, u_{h_{k}}^{*(\ell+1)}\right)$ has the following error estimates

$$
\begin{aligned}
\left\|\widehat{u}_{h_{k}}-u_{h_{k}}^{(\ell+1)}\right\|_{1} & \leq \gamma\left\|\widehat{u}_{h_{k}}-u_{h_{k}}^{(\ell)}\right\|_{1}, \\
\left\|\widehat{u}_{h_{k}}^{*}-u_{h_{k}}^{(\ell+1)}\right\|_{1} & \leq \gamma^{*}\left\|\widehat{u}_{h_{k}}^{*}-u_{h_{k}}^{*(\ell)}\right\|_{1}, \\
\left\|\widehat{u}_{h_{k}}-u_{h_{k}}^{(\ell+1)}\right\|_{0} & \leq C_{\lambda} \eta_{a}\left(V_{H}\right)\left\|\widehat{u}_{h_{k}}-u_{h_{k}}^{(\ell+1)}\right\|_{1}, \\
\left\|\widehat{u}_{h_{k}}^{*}-u_{h_{k}}^{*(\ell+1)}\right\|_{0} & \leq C_{\lambda} \eta_{a}^{*}\left(V_{H}\right)\left\|\widehat{u}_{h_{k}}^{*}-u_{h_{k}}^{*(\ell+1)}\right\|_{1}, \\
\left\|\widehat{u}_{h_{k}}-u_{h_{k}}^{(\ell+1)}\right\|_{-1 / 2, \partial \Omega} & \leq C_{\lambda} \eta_{a}\left(V_{H}\right)\left\|\widehat{u}_{h_{k}}-u_{h_{k}}^{(\ell+1)}\right\|_{1}, \\
\left\|\widehat{u}_{h_{k}}^{*}-u_{h_{k}}^{*(\ell+1)}\right\|_{-1 / 2, \partial \Omega} & \leq C_{\lambda} \eta_{a}^{*}\left(V_{H}\right)\left\|\widehat{u}_{h_{k}}^{*}-u_{h_{k}}^{*(\ell+1)}\right\|_{1}, \\
\left|\widehat{\lambda}_{h_{k}}-\lambda_{h_{k}}^{(\ell+1)}\right| & \leq C_{\lambda}\left\|\widehat{u}_{h_{k}}-u_{h_{k}}^{(\ell+1)}\right\|_{1}\left\|\widehat{u}_{h_{k}}^{*}-u_{h_{k}}^{*(\ell+1)}\right\|_{1},
\end{aligned}
$$

where

$$
\begin{array}{r}
\gamma=\theta+\left(\theta C_{\lambda}+(1+\theta) \widetilde{C}+(1+\theta) \widetilde{C} C_{\lambda}\left(\eta_{a}\left(V_{H}\right)+\delta\left(u^{*}, V_{H}\right)\right)\right)\left(\eta_{a}\left(V_{H}\right)+\delta\left(u^{*}, V_{H}\right)\right) \\
\gamma^{*}=\theta+\left(\theta C_{\lambda}+(1+\theta) \widetilde{C}^{*}+(1+\theta) \widetilde{C}^{*} C_{\lambda}\left(\eta_{a}^{*}\left(V_{H}\right)+\delta\left(u, V_{H}\right)\right)\right)\left(\eta_{a}^{*}\left(V_{H}\right)+\delta\left(u, V_{H}\right)\right)
\end{array}
$$

and

$$
\begin{aligned}
\widetilde{C} & =C_{a}^{2} \check{C}\left(C_{t r}\left(\left|\widehat{\lambda}_{h_{k}}\right|+\left\|u_{h_{k}}^{(\ell)}\right\|_{-1 / 2, \partial \Omega}\right)+\left\|1+\kappa^{2} n(x)\right\|_{\infty}\right), \\
\widetilde{C}^{*} & =C_{a}^{2} \check{C}\left(C_{t r}\left(\left|\widehat{\lambda}_{h_{k}}\right|+\left\|u_{h_{k}}^{*(\ell)}\right\|_{-1 / 2, \partial \Omega}\right)+\left\|1+\kappa^{2} \bar{n}(x)\right\|_{\infty}\right) .
\end{aligned}
$$

Proof. From (2.6) and (3.5) and trace theorem, setting $w_{h_{k}}=\widehat{u}_{h_{k}}-\check{u}_{h_{k}}^{(\ell+1)} \in V_{h_{k}}$, we have

$$
\begin{aligned}
a_{s}\left(\widehat{u}_{h_{k}}-\check{u}_{h_{k}}^{(\ell+1)}, w_{h_{k}}\right)=- & b\left(\widehat{\lambda}_{h_{k}} \widehat{u}_{h_{k}}-\lambda_{h_{k}}^{(\ell)} u_{h_{k}}^{(\ell)}, w_{h_{k}}\right)+\left(\left(1+\kappa^{2} n(x)\right)\left(\widehat{u}_{h_{k}}-u_{h_{k}}^{(\ell)}\right), w_{h_{k}}\right) \\
\leq & \left(\left|\widehat{\lambda}_{h_{k}}\right|\left\|\widehat{u}_{h_{k}}-u_{h_{k}}^{(\ell)}\right\|_{-1 / 2, \partial \Omega}+\left|\widehat{\lambda}_{h_{k}}-\lambda_{h_{k}}^{(\ell)}\right|\left\|u_{h_{k}}^{(\ell)}\right\|_{-1 / 2, \partial \Omega}\right)\left\|w_{h_{k}}\right\|_{1 / 2, \partial \Omega} \\
& +\left\|1+\kappa^{2} n(x)\right\|_{\infty}\left\|\widehat{u}_{h_{k}}-u_{h_{k}}^{(\ell)}\right\|_{0}\left\|w_{h_{k}}\right\|_{0} \\
\leq & \left(\left|\widehat{\lambda}_{h_{k}}\right|\left\|\widehat{u}_{h_{k}}-u_{h_{k}}^{(\ell)}\right\|_{-1 / 2, \partial \Omega}+\left|\widehat{\lambda}_{h_{k}}-\lambda_{h_{k}}^{(\ell)}\right|\left\|u_{h_{k}}^{(\ell)}\right\|_{-1 / 2, \partial \Omega}\right) C_{t r}\left\|w_{h_{k}}\right\|_{1} \\
& +\left\|1+\kappa^{2} n(x)\right\|_{\infty}\left\|\widehat{u}_{h_{k}}-u_{h_{k}}^{(\ell)}\right\|_{0}\left\|w_{h_{k}}\right\|_{1},
\end{aligned}
$$


where $C_{t r}$ is the constant in trace theorem. It leads to the following estimates by using (2.3), (3.10), (3.12) and $(3.14)$

$$
\left\|\widehat{u}_{h_{k}}-\check{u}_{h_{k}}^{(\ell+1)}\right\|_{1} \leq \widetilde{C}\left(\eta_{a}\left(V_{H}\right)+\delta\left(u^{*}, V_{H}\right)\right)\left\|\widehat{u}_{h_{k}}-u_{h_{k}}^{(\ell)}\right\|_{1}
$$

where $\widetilde{C}=C_{a}^{2} \check{C}\left(C_{t r}\left(\left|\widehat{\lambda}_{h_{k}}\right|+\left\|u_{h_{k}}^{(\ell)}\right\|_{-1 / 2, \partial \Omega}\right)+\left\|1+\kappa^{2} n(x)\right\|_{\infty}\right)$.

Combining (3.8) and (3.24) leads to the following error estimate for $\widetilde{u}_{h_{k}}^{(\ell+1)}$

$$
\begin{aligned}
\left\|\check{u}_{h_{k}}^{(\ell+1)}-\widetilde{u}_{h_{k}}^{(\ell+1)}\right\|_{1} & \leq \theta\left\|\check{u}_{h_{k}}^{(\ell+1)}-u_{h_{k}}^{(\ell)}\right\|_{1} \\
& \leq \theta\left(\left\|\check{u}_{h_{k}}^{(\ell+1)}-\widehat{u}_{h_{k}}\right\|_{1}+\left\|\widehat{u}_{h_{k}}-u_{h_{k}}^{(\ell)}\right\|_{1}\right) \\
& \leq \theta\left(1+\widetilde{C}\left(\eta_{a}\left(V_{H}\right)+\delta\left(u^{*}, V_{H}\right)\right)\right)\left\|\widehat{u}_{h_{k}}-u_{h_{k}}^{(\ell)}\right\|_{1} .
\end{aligned}
$$

Then from (3.24) and (3.25), we have the following inequalities

$$
\begin{aligned}
\left\|\widehat{u}_{h_{k}}-\widetilde{u}_{h_{k}}^{(\ell+1)}\right\|_{1} & \leq\left\|\widehat{u}_{h_{k}}-\check{u}_{h_{k}}^{(\ell+1)}\right\|_{1}+\left\|\check{u}_{h_{k}}^{(\ell+1)}-\widetilde{u}_{h_{k}}^{(\ell+1)}\right\|_{1} \\
& \leq\left(\theta+(1+\theta) \widetilde{C}\left(\eta_{a}\left(V_{H}\right)+\delta\left(u^{*}, V_{H}\right)\right)\right)\left\|\widehat{u}_{h_{k}}-u_{h_{k}}^{(\ell)}\right\|_{1} .
\end{aligned}
$$

Since $V_{H, h_{k}} \subset V_{h_{k}}$, the eigenvalue problem (3.7) can be regarded as a finite dimensional subspace approximation of the eigenvalue problem (2.6). Combining $V_{H} \subset V_{H, h_{k}}$ and (2.12) the following estimates hold

$$
\begin{aligned}
\left\|\widehat{u}_{h_{k}}-u_{h_{k}}^{(\ell+1)}\right\|_{1} & \leq\left(1+C_{\lambda}\left(\eta_{a}\left(V_{H, h_{k}}\right)+\delta\left(u^{*}, V_{H, h_{k}}\right)\right)\right) \inf _{v_{H, h_{k}} \in V_{H, h_{k}}}\left\|\widehat{u}_{h_{k}}-v_{H, h_{k}}\right\|_{1} \\
& \leq\left(1+C_{\lambda}\left(\eta_{a}\left(V_{H}\right)+\delta\left(u^{*}, V_{H}\right)\right)\right)\left\|\widehat{u}_{h_{k}}-\widetilde{u}_{h_{k}}^{(\ell+1)}\right\|_{1} \\
& \leq \gamma\left\|\widehat{u}_{h_{k}}-u_{h_{k}}^{(\ell)}\right\|_{1},
\end{aligned}
$$

here $\gamma=\theta+\left(\theta C_{\lambda}+(1+\theta) \widetilde{C}+(1+\theta) \widetilde{C} C_{\lambda}\left(\eta_{a}\left(V_{H}\right)+\delta\left(u^{*}, V_{H}\right)\right)\right)\left(\eta_{a}\left(V_{H}\right)+\delta\left(u^{*}, V_{H}\right)\right)$. That is the desired result (3.15).

Using (2.14), we have the following estimates

$$
\begin{aligned}
\left\|\widehat{u}_{h_{k}}-u_{h_{k}}^{(\ell+1)}\right\|_{0} & \leq C_{\lambda} \eta_{a}\left(V_{H, h_{k}}\right) \inf _{v_{H, h_{k}} \in V_{H, h_{k}}}\left\|\widehat{u}_{h_{k}}-v_{H, h_{k}}\right\|_{1} \\
& \leq C_{\lambda} \eta_{a}\left(V_{H}\right)\left\|\widehat{u}_{h_{k}}-u_{h_{k}}^{(\ell+1)}\right\|_{1} .
\end{aligned}
$$

Similarly, using (2.16), we have

$$
\begin{aligned}
\left\|\widehat{u}_{h_{k}}-u_{h_{k}}^{(\ell+1)}\right\|_{-1 / 2, \partial \Omega} & \leq C_{\lambda} \eta_{a}\left(V_{H, h_{k}}\right) \inf _{v_{H, h_{k}} \in V_{H, h_{k}}}\left\|\widehat{u}_{h_{k}}-v_{H, h_{k}}\right\|_{1} \\
& \leq C_{\lambda} \eta_{a}\left(V_{H}\right)\left\|\widehat{u}_{h_{k}}-u_{h_{k}}^{(\ell+1)}\right\|_{1} .
\end{aligned}
$$

The estimates (3.16), (3.18) and (3.20) for the adjoint problem can be proved in the similar way. Then the desired (3.21) is the direct result of Lemma 2.2 and 2.6.

Remark 3.4. Definitions (3.22) and (3.23), Lemma 2.2, Corollary 2.3 together with Lemma 3.2 imply that $\gamma<1$ and $\gamma^{*}<1$ when $H$ is small enough. If the considering eigenvalue $\lambda$ is large or the spectral gap is small, then we need to choose a smaller $H$. Furthermore, we can increase the multigrid steps for boundary value problem to reduce $\theta$, and then makes $\gamma$ and $\gamma^{*}$ smaller. However, the practical application is not limited by these requirements. Actually, $H$ and the coarsest space only need to match the number of eigenpairs to be computed. In numerical implementations, $H$ does not need to be very small (e.g. $H=\frac{\sqrt{2}}{8}$ in Subsect. 5.1). 


\subsection{Full multigrid method for non-selfadjoint Steklov eigenvalue problem}

Based on the one correction step defined in Algorithm 3.1, a type of full multigrid scheme will be introduced in this subsection. The optimal error estimate with the optimal computational work will be deduced for this type of full multigrid method.

Since the multigrid method for the boundary value problem has the uniform error reduction rate (cf. [20]), we can choose suitable $m$ such that $\theta<1$ in (3.8) and (3.9). From the definition (3.22) for $\gamma$, it is obvious that $\gamma<1$ if the mesh size $H$ of $\mathcal{T}_{H}$ is small enough. Based on these property, we can design a full multigrid method for non-selfadjoint Steklov eigenvalue problem as follows.

\section{Algorithm 3.2. Full Multigrid Scheme}

1. Solve the following non-selfadjoint Steklov eigenvalue problem in $V_{h_{1}}$ :

Find $\left(\lambda_{h_{1}}, u_{h_{1}}\right) \in \mathbb{C} \times V_{h_{1}} \times V_{h_{1}}$ such that

$$
a\left(u_{h_{1}}, v_{h_{1}}\right)=-\lambda_{h_{1}} b\left(u_{h_{1}}, v_{h_{1}}\right), \quad \forall v_{h_{1}} \in V_{h_{1}} .
$$

and find $u_{h_{1}}^{*} \in V_{h_{1}}$ according to Lemma 2.4 and Remark 2.5.

Hence we get the desired eigenpair approximation $\left(\lambda_{h_{1}}, u_{h_{1}}, u_{h_{1}}^{*}\right) \in \mathbb{C} \times V_{h_{1}} \times V_{h_{1}}$.

2. For $k=2, \cdots, n-1$, do the following iterations

- Set $\lambda_{h_{k}}^{(0)}=\lambda_{h_{k-1}}, u_{h_{k}}^{(0)}=u_{h_{k-1}}$ and $u_{h_{k}}^{*(0)}=u_{h_{k-1}}^{*}$.

- Perform the correction steps

$$
\left(\lambda_{h_{k}}^{(\ell+1)}, u_{h_{k}}^{(\ell+1)}, u_{h_{k}}^{*(\ell+1)}\right)=\operatorname{EigenMG}\left(V_{H}, \lambda_{h_{k}}^{(\ell)}, u_{h_{k}}^{(\ell)}, u_{h_{k}}^{*(\ell)}, V_{h_{k}}, m\right), \quad \text { for } \ell=0, \cdots, p-1 .
$$

- Set $\lambda_{h_{k}}=\lambda_{h_{k}}^{(p)}, u_{h_{k}}=u_{h_{k}}^{(p)}$ and $u_{h_{k}}^{*}=u_{h_{k}}^{*(p)}$.

End Do

3. Do the following iterations on the finest level space $V_{h_{n}}$

- Set $\lambda_{h_{n}}^{(0)}=\lambda_{h_{n-1}}, u_{h_{n}}^{(0)}=u_{h_{n-1}}$ and $u_{h_{n}}^{*(0)}=u_{h_{n-1}}^{*}$.

- Perform the correction steps

$$
\left(\lambda_{h_{n}}^{(\ell+1)}, u_{h_{n}}^{(\ell+1)}, u_{h_{n}}^{*(\ell+1)}\right)=\operatorname{EigenMG}\left(V_{H}, \lambda_{h_{n}}^{(\ell)}, u_{h_{n}}^{(\ell)}, u_{h_{n}}^{*(\ell)}, V_{h_{n}}, m\right), \quad \text { for } \ell=0, \cdots, p-1 .
$$

- Set $u_{h_{n}}=u_{h_{n}}^{(p)}$ and $u_{h_{n}}^{*}=u_{h_{n}}^{*(p)}$.

- Compute the generalized Rayleigh quotient

$$
\lambda_{h_{n}}=\frac{a\left(u_{h_{n}}, u_{h_{n}}^{*}\right)}{b\left(u_{h_{n}}, u_{h_{n}}^{*}\right)} .
$$

Finally, we obtain an eigenpair approximation $\left(\lambda_{h_{n}}, u_{h_{n}}, u_{h_{n}}^{*}\right) \in \mathbb{C} \times V_{h_{n}} \times V_{h_{n}}$ in the finest space.

Remark 3.5. Actually, if we know the distribution of eigenvalues in advance, our algorithm (Algorithm 3.2, 3.4 and 4.2) can solve any eigenvalue we want. But, the distribution of eigenvalues is an open problem. So we just show the convergence of several eigenvalues with smallest magnitude in our numerical tests. 
Theorem 3.6. Assume (3.3) and the conditions of Theorem 3.3 hold. After implementing Algorithm 3.2, the resultant eigenpair approximation $\left(\lambda_{h_{n}}, u_{h_{n}}, u_{h_{n}}^{*}\right)$ has the following error estimate

$$
\begin{aligned}
\left\|\widehat{u}_{h_{n}}-u_{h_{n}}\right\|_{1} & \leq 2 C_{\lambda} \frac{C_{\delta} \beta^{\zeta} \gamma^{p}}{1-C_{\delta} \beta^{\zeta} \gamma^{p}} \delta\left(u, V_{h_{n}}\right), \\
\left\|\widehat{u}_{h_{n}}^{*}-u_{h_{n}}^{*}\right\|_{1} & \leq 2 C_{\lambda} \frac{C_{\delta}^{*} \beta^{\zeta}\left(\gamma^{*}\right)^{p}}{1-C_{\delta}^{*} \beta^{\zeta}\left(\gamma^{*}\right)^{p}} \delta\left(u^{*}, V_{h_{n}}\right), \\
\left\|\widehat{u}_{h_{n}}-u_{h_{n}}\right\|_{-1 / 2, \partial \Omega} & \leq 2 C_{\lambda} \frac{C_{\delta} \beta^{\zeta} \gamma^{p}}{1-C_{\delta} \beta^{\zeta} \gamma^{p}} \eta_{a}\left(V_{H}\right) \delta\left(u, V_{h_{n}}\right), \\
\left\|\widehat{u}_{h_{n}}^{*}-u_{h_{n}}^{*}\right\|_{-1 / 2, \partial \Omega} & \leq 2 C_{\lambda} \frac{C_{\delta}^{*} \beta^{\zeta}\left(\gamma^{*}\right)^{p}}{1-C_{\delta}^{*} \beta^{\zeta}\left(\gamma^{*}\right)^{p}} \eta_{a}^{*}\left(V_{H}\right) \delta\left(u^{*}, V_{h_{n}}\right), \\
\left|\widehat{\lambda}_{h_{n}}-\lambda_{h_{n}}\right| & \leq 4 C_{\lambda}^{2} \frac{C_{\delta} C_{\delta}^{*} \beta^{2 \zeta}\left(\gamma \gamma^{*}\right)^{p}}{\left(1-C_{\delta} \beta^{\zeta} \gamma^{p}\right)\left(1-C_{\delta}^{*} \beta^{\zeta}\left(\gamma^{*}\right)^{p}\right)} \delta\left(u, V_{h_{n}}\right) \delta\left(u^{*}, V_{h_{n}}\right),
\end{aligned}
$$

under the conditions $C_{\delta} \beta^{\zeta} \gamma^{p}<1$ and $C_{\delta}^{*} \beta^{\zeta}\left(\gamma^{*}\right)^{p}<1$.

Proof. Define $e_{k}:=\widehat{u}_{h_{k}}-u_{h_{k}}$. Then from Step 1 in Algorithm 3.2, we have $e_{1}=0$. Then the assumption (3.10)-(3.14) in Theorem 3.3 are satisfied for $k=1$. From Algorithms 3.1 and 3.2, Theorem 3.3, and recursive argument, the assumption (3.10)-(3.14) hold for each level of space $V_{h_{k}}(k=1, \cdots, n)$. Then the convergence rate (3.15) and (3.16) are valid for all $k=1, \cdots, n$ and $\ell=0, \cdots, p-1$.

For $k=2, \cdots, n$, from Lemma 2.2 and 3.3 , and recursive argument, we have

$$
\begin{aligned}
\left\|e_{k}\right\|_{1} & \leq \gamma^{p}\left\|\widehat{u}_{h_{k}}-u_{h_{k-1}}\right\|_{1} \\
& \leq \gamma^{p}\left(\left\|\widehat{u}_{h_{k}}-\widehat{u}_{h_{k-1}}\right\|_{1}+\left\|\widehat{u}_{h_{k-1}}-u_{h_{k-1}}\right\|_{1}\right) \\
& \leq \gamma^{p}\left(\left\|\widehat{u}_{h_{k}}-u\right\|_{1}+\left\|u-\widehat{u}_{h_{k-1}}\right\|_{1}+\left\|\widehat{u}_{h_{k-1}}-u_{h_{k-1}}\right\|_{1}\right) \\
& \leq \gamma^{p}\left(C_{\lambda} \delta\left(u, V_{h_{k}}\right)+C_{\lambda} \delta\left(u, V_{h_{k-1}}\right)+\left\|e_{k-1}\right\|_{1}\right) \\
& \leq \gamma^{p}\left(2 C_{\lambda} \delta\left(u, V_{h_{k-1}}\right)+\left\|e_{k-1}\right\|_{1}\right) .
\end{aligned}
$$

From (3.3), we have the following relationship

$$
\delta\left(u, V_{h_{k}}\right) \leq\left(C_{\delta} \beta^{\zeta}\right)^{n-k} \delta\left(u, V_{h_{n}}\right) .
$$

Then, by iterating inequality (3.36) and the condition $C_{\delta} \beta^{\zeta} \gamma^{p}<1$, the following inequalities hold

$$
\begin{aligned}
\left\|e_{n}\right\|_{1} & \leq 2 C_{\lambda} \gamma^{p} \delta\left(u, V_{h_{n-1}}\right)+2 C_{\lambda} \gamma^{2 p} \delta\left(u, V_{h_{n-2}}\right)+\cdots+2 C_{\lambda} \gamma^{(n-1) p} \delta\left(u, V_{h_{1}}\right) \\
& \leq 2 C_{\lambda} \sum_{k=1}^{n-1} \gamma^{(n-k) p} \delta\left(u, V_{h_{k}}\right)=2 C_{\lambda}\left(\sum_{k=1}^{n-1}\left(C_{\delta} \beta^{\zeta} \gamma^{p}\right)^{n-k}\right) \delta\left(u, V_{h_{n}}\right) \\
& \leq 2 C_{\lambda} \frac{C_{\delta} \beta^{\zeta} \gamma^{p}}{1-C_{\delta} \beta^{\zeta} \gamma^{p}} \delta\left(u, V_{h_{n}}\right) .
\end{aligned}
$$

For such choice of $p$, we arrive the desired result (3.31). Equation (3.33) is obtained by (3.19) and (3.31). Equation (3.32) and (3.34) can be proved in the similar way. Furthermore, (3.35) can be obtained similar to (3.21) from Lemma 2.6.

Now, we turn to the following final error estimates for our full multigrid method. 
Theorem 3.7. Assume the conditions of Lemma 2.2, Corollary 2.3 and Theorem 3.6 hold. After implementing Algorithm 3.2, the resultant eigenpair approximation $\left(\lambda_{h_{n}}, u_{h_{n}}, u_{h_{n}}^{*}\right)$ has the following error estimates

$$
\begin{aligned}
\left\|u-u_{h_{n}}\right\|_{1} & \leq C_{1} h_{n}^{\sigma}, \\
\left\|u^{*}-u_{h_{n}}^{*}\right\|_{1} & \leq C_{2} h_{n}^{\sigma}, \\
\left\|u-u_{h_{n}}\right\|_{-1 / 2, \partial \Omega} & \leq C_{3} H^{\sigma} h_{n}^{\sigma}, \\
\left\|u^{*}-u_{h_{n}}^{*}\right\|_{-1 / 2, \partial \Omega} & \leq C_{4} H^{\sigma} h_{n}^{\sigma}, \\
\left|\lambda-\lambda_{h_{n}}\right| & \leq C_{5} h_{n}^{2 \sigma},
\end{aligned}
$$

where $C_{1}=\left(1+2 C_{\lambda} C_{\sigma} h_{n}^{\sigma}+2 C_{\lambda} \frac{C_{\delta} \beta^{\zeta} \gamma^{p}}{1-C_{\delta} \beta^{\zeta} \gamma^{p}}\right) C_{\sigma}, C_{2}=\left(1+2 C_{\lambda} C_{\sigma} h_{n}^{\sigma}+2 C_{\lambda} \frac{C_{\delta}^{*} \beta^{\zeta}\left(\gamma^{*}\right)^{p}}{1-C_{\delta}^{*} \beta^{\zeta}\left(\gamma^{*}\right)^{p}}\right) C_{\sigma}$, $C_{3}=\left(C_{\lambda} C_{\sigma}+2 C_{\lambda} C_{\sigma} \frac{C_{\delta} \beta^{\zeta} \gamma^{p}}{1-C_{\delta} \beta^{\zeta} \gamma^{p}}\right) C_{\sigma}, C_{4}=\left(C_{\lambda} C_{\sigma}+2 C_{\lambda} C_{\sigma} \frac{C_{\delta}^{*} \beta^{\zeta}\left(\gamma^{*}\right)^{p}}{1-C_{\delta}^{*} \beta^{\zeta}\left(\gamma^{*}\right)^{p}}\right) C_{\sigma}$ and $C_{5}=C_{1} C_{2} \frac{\left|\lambda_{h_{n}}\right|}{C_{0}}\left(C_{a}^{2}+\left\|1+\kappa^{2} n(x)\right\|_{\infty}+|\lambda| C_{t r}^{2}\right)$.

Proof. Based on (2.12), (3.31), Corollary 2.3 and triangular inequality, we have

$$
\begin{aligned}
\left\|u-u_{h_{n}}\right\|_{1} & \leq\left\|u-\widehat{u}_{h_{n}}\right\|_{1}+\left\|\widehat{u}_{h_{n}}-u_{h_{n}}\right\|_{1} \\
& \leq\left(1+C_{\lambda}\left(\eta_{a}\left(V_{h_{n}}\right)+\delta\left(u^{*}, V_{h_{n}}\right)\right)\right) \delta\left(u, V_{h_{n}}\right)+2 C_{\lambda} \frac{C_{\delta} \beta^{\zeta} \gamma^{p}}{1-C_{\delta} \beta^{\zeta} \gamma^{p}} \delta\left(u, V_{h_{n}}\right) \\
& \leq\left(1+C_{\lambda}\left(\eta_{a}\left(V_{h_{n}}\right)+\delta\left(u^{*}, V_{h_{n}}\right)\right)+2 C_{\lambda} \frac{C_{\delta} \beta^{\zeta} \gamma^{p}}{1-C_{\delta} \beta^{\zeta} \gamma^{p}}\right) \delta\left(u, V_{h_{n}}\right) \\
& \leq\left(1+2 C_{\lambda} C_{\sigma} h_{n}^{\sigma}+2 C_{\lambda} \frac{C_{\delta} \beta^{\zeta} \gamma^{p}}{1-C_{\delta} \beta^{\zeta} \gamma^{p}}\right) C_{\sigma} h_{n}^{\sigma} .
\end{aligned}
$$

This is the desired result (3.39).

From (2.16), (3.33), Corollary 2.3 and triangular inequality, the following estimates hold:

$$
\begin{aligned}
\left\|u-u_{h_{n}}\right\|_{-1 / 2, \partial \Omega} & \leq\left\|u-\widehat{u}_{h_{n}}\right\|_{-1 / 2, \partial \Omega}+\left\|\widehat{u}_{h_{n}}-u_{h_{n}}\right\|_{-1 / 2, \partial \Omega} \\
& \leq C_{\lambda} \eta_{a}\left(V_{h_{n}}\right) \delta\left(u, V_{h_{n}}\right)+2 C_{\lambda} \frac{C_{\delta} \beta^{\zeta} \gamma^{p}}{1-C_{\delta} \beta^{\zeta} \gamma^{p}} \eta_{a}\left(V_{H}\right) \delta\left(u, V_{h_{n}}\right) \\
& \leq\left(C_{\lambda} \eta_{a}\left(V_{h_{n}}\right)+2 C_{\lambda} \frac{C_{\delta} \beta^{\zeta} \gamma^{p}}{1-C_{\delta} \beta^{\zeta} \gamma^{p}} \eta_{a}\left(V_{H}\right)\right) \delta\left(u, V_{h_{n}}\right) \\
& \leq\left(C_{\lambda} C_{\sigma} h_{n}^{\sigma}+2 C_{\lambda} C_{\sigma} \frac{C_{\delta} \beta^{\zeta} \gamma^{p}}{1-C_{\delta} \beta^{\zeta} \gamma^{p}} H^{\sigma}\right) C_{\sigma} h_{n}^{\sigma} \\
& \leq\left(C_{\lambda} C_{\sigma}+2 C_{\lambda} C_{\sigma} \frac{C_{\delta} \beta^{\zeta} \gamma^{p}}{1-C_{\delta} \beta^{\zeta} \gamma^{p}}\right) C_{\sigma} H^{\sigma} h_{n}^{\sigma} .
\end{aligned}
$$

This is the desired result (3.41).

The estimates (3.40) and (3.42) for the adjoint problem can be proved in the similar way. 
Combining Lemma 2.4, 2.6, (2.3), (3.30) and trace theorem, we obtain

$$
\begin{aligned}
\left|\lambda_{h_{n}}-\lambda\right| & =\left|\frac{-a\left(u_{h_{n}}-u, u_{h_{n}}^{*}-u^{*}\right)-\lambda b\left(u_{h_{n}}-u, u_{h_{n}}^{*}-u^{*}\right)}{b\left(u_{h_{n}}, u_{h_{n}}^{*}\right)}\right| \\
& \leq \frac{\left|a_{s}\left(u_{h_{n}}-u, u_{h_{n}}^{*}-u^{*}\right)\right|+\left|\left(\left(1+\kappa^{2} n(x)\right)\left(u_{h_{n}}-u\right), u_{h_{n}}^{*}-u^{*}\right)\right|+|\lambda|\left|b\left(u_{h_{n}}-u, u_{h_{n}}^{*}-u^{*}\right)\right|}{\left|b\left(u_{h_{n}}, u_{h_{n}}^{*}\right)\right|} \\
& \leq \frac{\left|\lambda_{h_{n}}\right|}{C_{0}}\left(C_{a}^{2}\left\|u_{h_{n}}-u\right\|_{1}\left\|u_{h_{n}}^{*}-u^{*}\right\|_{1}+\left\|1+\kappa^{2} n(x)\right\|_{\infty} \| u_{h_{n}}-u\right)\left\|_{1}\right\| u_{h_{n}}^{*}-u^{*} \|_{1} \\
\left.\quad+|\lambda| C_{t r}^{2}\left\|u_{h_{n}}-u\right\|_{1}\left\|u_{h_{n}}^{*}-u^{*}\right\|_{1}\right) & \\
\leq & \frac{\left|\lambda_{h_{n}}\right|}{C_{0}}\left(C_{a}^{2}+\left\|1+\kappa^{2} n(x)\right\|_{\infty}+|\lambda| C_{t r}^{2}\right)\left\|u_{h_{n}}-u\right\|_{1}\left\|u_{h_{n}}^{*}-u^{*}\right\|_{1} .
\end{aligned}
$$

From (3.44), (3.39) and (3.40), we can get the desired result (3.43).

\subsection{Estimate of the computational work}

In this subsection, we turn our attention to the estimate of computational work for the full multigrid method defined in Algorithm 3.2. It will be shown that the full multigrid method makes solving the non-selfadjoint Steklov eigenvalue problem need almost the same work as solving the corresponding linear boundary value problems. Besides, we turn our attention to the estimate of computational work for the full multigrid method defined in Algorithm 3.2.

First, we define the dimension of each level finite element space as $N_{k}:=\operatorname{dim} V_{h_{k}}$. Then we have

$$
N_{k} \approx\left(\frac{1}{\beta}\right)^{d(n-k) \zeta} N_{n}, \quad k=1,2, \cdots, n .
$$

Theorem 3.8. Assume the eigenvalue solving in the coarse spaces $V_{H, h_{k}}(k=1, \cdots, n)$ and $V_{h_{1}}$ need work $M_{H}$ and $M_{h_{1}}$, respectively, and the work of the multigrid solver in each level space $V_{h_{k}}$ is $\mathcal{O}\left(N_{k}\right)$ for $k=$ $2,3, \cdots, n$. Then the total computational work of Algorithm 3.2 can be bounded by $\mathcal{O}\left(N_{n}+M_{H} \log N_{n}+M_{h_{1}}\right)$, and furthermore $\mathcal{O}\left(N_{n}\right)$ provided $M_{H} \ll N_{n}$ and $M_{h_{1}} \leq N_{n}$.

Proof. We use $W_{k}$ to denote the work involved in each correction step on the $k$-th finite element space $V_{h_{k}}$.

Based on Algorithms 3.1 and 3.2,

$$
W_{1}=2 M_{h_{1}}, \quad W_{k} \lesssim 2 M_{H}+2 m N_{k}, k=2, \cdots, n .
$$

Based on the property (3.45), iterating (3.46) leads to

$$
\begin{aligned}
\text { Total work } & =W_{1}+\sum_{k=2}^{n}\left(p W_{k}\right) \lesssim 2 M_{h_{1}}+\sum_{k=2}^{n}\left(2 p M_{H}+2 m p N_{k}\right) \\
& \lesssim M_{h_{1}}+n M_{H}+\sum_{k=2}^{n} N_{k} \\
& \lesssim M_{h_{1}}+M_{H} \log N_{n}+\sum_{k=2}^{n}\left(\frac{1}{\beta}\right)^{d(n-k) \zeta} N_{n} \\
& \lesssim N_{n}+M_{H} \log N_{n}+M_{h_{1}} .
\end{aligned}
$$

This is the desired result and we complete the proof. 
Remark 3.9. The high efficiency of the multigrid method for boundary value problems leads to that one does not need to choose large $m$ and $p$, please see Section 5 and $[20,26]$. The computational works $\mathcal{O}\left(M_{H}\right)$ and $\mathcal{O}\left(M_{h_{1}}\right)$ for the non-selfadjoint Steklov eigenvalue problem and its adjoint problem depend on the eigenvalue solver. Fortunately, they are very small since the eigenvalue problems which are required to solve are defined on very low dimensional spaces $V_{H, h_{k}}(k=2, \cdots, n)$ and $V_{h_{1}}$. Thus, Algorithm 3.2 has the qusi-optimal complexity.

\subsection{Full multigrid method for computing multiple eigenpairs}

Based on full mutigrid method, we can extend Algorithm 3.2 to compute multiple eigenpairs. Firstly, we should introduce the one correction step for computing multiple eigenpairs of non-selfadjoint Steklov problem.

Assume that we have obtained the $q$ eigenpair approximations $\left(\lambda_{j, h_{k}}^{(\ell)}, u_{j, h_{k}}^{(\ell)}, u_{j, h_{k}}^{*(\ell)}\right) \in \mathbb{C} \times V_{h_{k}} \times V_{h_{k}}$ for $j=i, \cdots, i+q-1$. Now we introduce a type of iteration step to improve the accuracy of the current eigenpair approximation $\left\{\lambda_{j, h_{k}}^{(\ell)}, u_{j, h_{k}}^{(\ell)}, u_{j, h_{k}}^{*(\ell)}\right\}_{j=i}^{i+q-1}$.

\section{Algorithm 3.3. One Correction Step for Computing Multiple Eigenpairs}

1. For $j=i, \cdots, i+q-1$, do:

Define the following auxiliary boundary value problems:

Find $\check{u}_{j, h_{k}}^{(\ell+1)} \in V_{h_{k}}$ such that

$$
a_{s}\left(\check{u}_{j, h_{k}}^{(\ell+1)}, v_{h_{k}}\right)=-\lambda_{j, h_{k}}^{(\ell)} b\left(u_{j, h_{k}}^{(\ell)}, v_{h_{k}}\right)+\left(\left(1+\kappa^{2} n(x)\right) u_{j, h_{k}}^{(\ell)}, v_{h_{k}}\right), \quad \forall v_{h_{k}} \in V_{h_{k}} .
$$

Find $\check{u}_{j, h_{k}}^{*(\ell+1)} \in V_{h_{k}}$ such that

$$
a_{s}\left(v_{h_{k}}, \check{u}_{j, h_{k}}^{*(\ell+1)}\right)=-\lambda_{j, h_{k}}^{(\ell)} b\left(v_{h_{k}}, u_{j, h_{k}}^{*(\ell)}\right)+\left(v_{h_{k}},\left(1+\kappa^{2} \bar{n}(x)\right) u_{j, h_{k}}^{*(\ell)}\right), \quad \forall v_{h_{k}} \in V_{h_{k}} .
$$

Solve (3.47) and (3.48) by performing m multigrid iteration steps with the initial guess value $u_{j, h_{k}}^{(\ell)}$ and $u_{j, h_{k}}^{*(\ell)}$ to obtain two new approximate solutions $\widetilde{u}_{j, h_{k}}^{(\ell+1)}$ and $\widetilde{u}_{j, h_{k}}^{*(\ell+1)}$, respectively.

2. Define

$$
\widetilde{V}_{H, h_{k}}=V_{H}+\operatorname{span}\left\{\widetilde{u}_{i, h_{k}}^{(\ell+1)}, \cdots, \widetilde{u}_{i+q-1, h_{k}}^{(\ell+1)}\right\}, \widetilde{V}_{H, h_{k}}^{*}=V_{H}+\operatorname{span}\left\{\widetilde{u}_{i, h_{k}}^{*(\ell+1)}, \cdots, \widetilde{u}_{i+q-1, h_{k}}^{*(\ell+1)}\right\},
$$

and solve the following eigenvalue problems for $j=i, \cdots, i+q-1$ :

Find $\left(\lambda_{j, h_{k}}^{(\ell+1)}, u_{j, h_{k}}^{(\ell+1)}\right) \in \mathbb{C} \times \widetilde{V}_{H, h_{k}}$ such that

$$
a\left(u_{j, h_{k}}^{(\ell+1)}, v_{H, h_{k}}\right)=-\lambda_{j, h_{k}}^{(\ell+1)} b\left(u_{j, h_{k}}^{(\ell+1)}, v_{H, h_{k}}\right), \quad \forall v_{H, h_{k}} \in \widetilde{V}_{H, h_{k}} .
$$

Find $u_{j, h_{k}}^{*(\ell+1)} \in \widetilde{V}_{H, h_{k}}^{*}$ according to Lemma 2.4 and Remark 2.5.

In order to simplify the notation and summarize the above two steps, we define

$$
\left\{\lambda_{j, h_{k}}^{(\ell+1)}, u_{j, h_{k}}^{(\ell+1)}, u_{j, h_{k}}^{*(\ell+1)}\right\}_{j=i}^{i+q-1}=\text { EigenMG_Multi }\left(V_{H},\left\{\lambda_{j, h_{k}}^{(\ell)}, u_{j, h_{k}}^{(\ell)}, u_{j, h_{k}}^{*(\ell)}\right\}_{j=i}^{i+q-1}, V_{h_{k}}, m\right) .
$$

Since using the multigrid method for solving the boundary value problems in Step 1 of Algorithm 3.3, we have the same uniform contraction rate as Lemma 3.2:

$$
\begin{gathered}
\left\|\check{u}_{j, h_{k}}^{(\ell+1)}-\widetilde{u}_{j, h_{k}}^{(\ell+1)}\right\|_{1} \leq \theta\left\|\check{u}_{j, h_{k}}^{(\ell+1)}-u_{j, h_{k}}^{(\ell)}\right\|_{1}, \\
\left\|\check{u}_{j, h_{k}}^{*(\ell+1)}-\widetilde{u}_{j, h_{k}}^{*(\ell+1)}\right\|_{1} \leq \theta\left\|\check{u}_{j, h_{k}}^{*(\ell+1)}-u_{j, h_{k}}^{*(\ell)}\right\|_{1} .
\end{gathered}
$$


Here we can choose suitable $m$ such that $\theta<1$ in the first step in Algorithm 3.3. Similar to Algorithm 3.2, we can build the following full multigrid method for several eigenvalue problems.

\section{Algorithm 3.4. Full Multigrid Scheme for Computing Multiple Eigenpairs}

1. Solve the following non-selfadjoint Steklov eigenvalue problems in $V_{h_{1}}$ : Find $\left\{\lambda_{j, h_{1}}, u_{j, h_{1}}\right\}_{j=i}^{i+q-1} \in \mathbb{C} \times V_{h_{1}}$ such that

$$
a\left(u_{j, h_{1}}, v_{h_{1}}\right)=-\lambda_{j, h_{1}} b\left(u_{j, h_{1}}, v_{h_{1}}\right), \quad \forall v_{h_{1}} \in V_{h_{1}} .
$$

Find $\left\{u_{j, h_{1}}^{*}\right\}_{j=i}^{i+q-1} \in V_{h_{1}}$ according to Lemma 2.4 and Remark 2.5.

Obtain the desired eigenpair approximation $\left\{\lambda_{j, h_{1}}, u_{j, h_{1}}, u_{j, h_{1}}^{*}\right\}_{j=i}^{i+q-1} \in \mathbb{C} \times V_{h_{1}} \times V_{h_{1}}$.

2. For $k=2, \cdots, n$, do the following iterations

- Set $\left\{\lambda_{j, h_{k}}^{(0)}, u_{j, h_{k}}^{(0)}, u_{j, h_{k}}^{*(0)}\right\}_{j=i}^{i+q-1}=\left\{\lambda_{j, h_{k-1}}, u_{j, h_{k-1}}, u_{j, h_{k-1}}^{*}\right\}_{j=i}^{i+q-1}$.

- Do the following one correction step by multigrid for $\ell=0, \cdots, p-1$ :

$$
\left\{\lambda_{j, h_{k}}^{(\ell+1)}, u_{j, h_{k}}^{(\ell+1)}, u_{j, h_{k}}^{*(\ell+1)}\right\}_{j=i}^{i+q-1}=\text { EigenMG_Multi }\left(V_{H},\left\{\lambda_{j, h_{k}}^{(\ell)}, u_{j, h_{k}}^{(\ell)}, u_{j, h_{k}}^{*(\ell)}\right\}_{j=i}^{i+q-1}, V_{h_{k}}, m\right) .
$$

- Set $\left\{\lambda_{j, h_{k}}, u_{j, h_{k}}, u_{j, h_{k}}^{*}\right\}_{j=i}^{i+q-1}=\left\{\lambda_{j, h_{k}}^{(p)}, u_{j, h_{k}}^{(p)}, u_{j, h_{k}}^{*(p)}\right\}_{j=i}^{i+q-1}$.

End Do

3. Do the following iterations on the finest level space $V_{h_{n}}$

- Set $\left\{\lambda_{j, h_{k}}^{(0)}, u_{j, h_{k}}^{(0)}, u_{j, h_{k}}^{*(0)}\right\}_{j=i}^{i+q-1}=\left\{\lambda_{j, h_{k-1}}, u_{j, h_{k-1}}, u_{j, h_{k-1}}^{*}\right\}_{j=i}^{i+q-1}$.

- Do the following one correction step by multigrid for $\ell=0, \cdots, p-1$ :

$$
\left\{\lambda_{j, h_{k}}^{(\ell+1)}, u_{j, h_{k}}^{(\ell+1)}, u_{j, h_{k}}^{*(\ell+1)}\right\}_{j=i}^{i+q-1}=\text { EigenMG_Multi }\left(V_{H},\left\{\lambda_{j, h_{k}}^{(\ell)}, u_{j, h_{k}}^{(\ell)}, u_{j, h_{k}}^{*(\ell)}\right\}_{j=i}^{i+q-1}, V_{h_{k}}, m\right) .
$$

- Set $\left\{u_{j, h_{k}}, u_{j, h_{k}}^{*}\right\}_{j=i}^{i+q-1}=\left\{u_{j, h_{k}}^{(p)}, u_{j, h_{k}}^{*(p)}\right\}_{j=i}^{i+q-1}$.

- Compute the generalized Rayleigh quotient

$$
\lambda_{j, h_{n}}=\frac{a\left(u_{j, h_{n}}, u_{j, h_{n}}^{*}\right)}{b\left(u_{j, h_{n}}, u_{j, h_{n}}^{*}\right)} \quad(j=i, \cdots, i+q-1) .
$$

Finally, we obtain an eigenpair approximation $\left\{\lambda_{j, h_{n}}, u_{j, h_{n}}, u_{j, h_{n}}^{*}\right\}_{j=i}^{i+q-1} \in \mathbb{C} \times V_{h_{n}} \times V_{h_{n}}$ in the finest space.

Remark 3.10. We can also obtain the optimal convergence order and almost optimal estimation of computation work of Algorithm 3.4 similar to Theorem 3.6 and Theorem 3.8. For more detail, please refer to [15].

\section{Adaptive full multigrid for multiple non-SElfadjoint Steklov eigenvalue PROBLEMS}

In this section, based on the a posteriori error estimators we will establish an adaptive full multigrid for the non-selfadjoint Steklov eigenvalue problem. Here, we only describe the scheme without analysis.

In the above full multigrid method, we refine the mesh uniformly. However, this is not practical since the amount of required memory will increase very rapidly as we refine the mesh. Hence, an efficient refinement strategy is desired. On the one hand, the solution should be resolved well with the refined mesh. On the other 
hand, the total amount of the mesh elements should be controlled well to make the simulation efficient. Based on the above discussion, adaptive mesh method is a competitive candidate for the refinement strategy.

A standard adaptive mesh process can be described by the following one

$$
\cdots \text { Solve } \rightarrow \text { Estimate } \rightarrow \text { Mark } \rightarrow \text { Refine } \cdots
$$

More precisely, to get $\mathcal{T}_{h_{k+1}}$ from $\mathcal{T}_{h_{k}}$, we first solve the discrete equation on $\mathcal{T}_{h_{k}}$ to get the approximate solution and then calculate the a posteriori error estimator on each mesh element. Next, we mark the elements with big errors and these elements are refined in such a way that the triangulation is still shape regular and conforming. Here, we choose the ZZ recovery-based error estimator [45,53] for (2.1). Based on the recovery operator $G_{h}$ (cf. $[45,53])$, for each element $K \in \mathcal{T}_{h}$, we define the local error indicator $\eta_{h}\left(u_{h}, K\right)$ and $\eta_{h}^{*}\left(u_{h}^{*}, K\right)$ by

$$
\eta_{h}\left(u_{h}, K\right):=\left\|G_{h} u_{h}-\nabla u_{h}\right\|_{0, K} \quad \text { and } \quad \eta_{h}^{*}\left(u_{h}^{*}, K\right):=\left\|G_{h} u_{h}^{*}-\nabla u_{h}^{*}\right\|_{0, K},
$$

and the error indicator for a subdomain $\omega \subset \Omega$ by

$$
\eta_{h}\left(u_{h}, \omega\right):=\left(\sum_{K \in \mathcal{T}_{h}, K \subset \omega} \eta_{h}^{2}\left(u_{h}, K\right)\right)^{1 / 2} \text { and } \eta_{h}^{*}\left(u_{h}^{*}, \omega\right):=\left(\sum_{K \in \mathcal{T}_{h}, K \subset \omega} \eta_{h}^{* 2}\left(u_{h}^{*}, K\right)\right)^{1 / 2}
$$

and the main error indicator for a subdomain $\omega \subset \Omega$ by

$$
\hat{\eta}_{h}\left(u_{h}, u_{h}^{*}, \omega\right):=\left(\eta_{h}^{2}\left(u_{h}, \omega\right)+\eta_{h}^{* 2}\left(u_{h}^{*}, \omega\right)\right)^{1 / 2}
$$

Based on the error indicator (4.2), we choose the Dörfler's marking strategy for $q$ approximations $\left\{\lambda_{j, h}, u_{j, h}, u_{j, h}^{*}\right\}_{j=i}^{i+q-1}$ to construct subset $\mathcal{M}_{h}$ for local refinement.

\section{Algorithm 4.1. Dörfler's Marking Strategy.}

1. Given a parameter $\widehat{\theta} \in(0,1)$.

2. Construct a minimal subset $\mathcal{M}_{h}$ from $\mathcal{T}_{h}$ by selecting some elements in $\mathcal{T}_{h}$ such that

$$
\sum_{j=i}^{i+q-1} \hat{\eta}_{h}\left(u_{j, h}, u_{j, h}^{*}, \mathcal{M}_{h}\right) \geq \widehat{\theta} \sum_{j=i}^{i+q-1} \hat{\eta}_{h}\left(u_{j, h}, u_{j, h}^{*}, \mathcal{T}_{h}\right) .
$$

3. Mark all the elements in $\mathcal{M}_{h}$.

Now we state the multigrid iteration scheme with adaptive method for non-selfadjoint Steklov eigenvalue problem. Based on the adaptive refinement method described above, and one correction step for multiple eigenvalues defined by Algorithm 3.3, the full multigrid method is given in the following algorithm. 
Algorithm 4.2. Full multigrid AFEM for multiple non-selfadjoint Steklov eigenvalue problems

1. Generate a coarse triangulation $\mathcal{T}_{H}$ on the computing domain $\Omega$, pick up an initial mesh $\mathcal{T}_{1}$ which is produced by refining $\mathcal{T}_{H}$ by the regular way, and choose a mark parameter $\hat{\theta} \in(0,1)$.

2. Build the initial finite element space $V_{h_{1}}$ on the triangulation $\mathcal{T}_{h_{1}}$, and solve the non-selfadjoint Steklov eigenvalue problem and its adjoint problem on the initial finite element space $V_{h_{1}}$ :

Find $\left(\lambda_{h_{1}}, u_{h_{1}}\right) \in \mathbb{C} \times V_{h_{1}}$ such that

$$
a\left(u_{h_{1}}, v_{h_{1}}\right)=-\lambda_{h_{1}} b\left(u_{h_{1}}, v_{h_{1}}\right), \quad \forall v_{h_{1}} \in V_{h_{1}} .
$$

Find $u_{h_{1}}^{*} \in V_{h_{1}}$ according to Lemma 2.4 and Remark 2.5.

Hence we obtain the desired eigenpair approximations $\left\{\lambda_{j, h_{1}}, u_{j, h_{1}}, u_{j, h_{1}}^{*}\right\}_{j=i}^{i+q-1} \in \mathbb{C} \times V_{h_{1}} \times V_{h_{1}}$, and set $k=1$.

3. Compute the local error indicators $\eta_{h}\left(u_{j, h_{k}}, K\right)$ and $\eta_{h}^{*}\left(u_{j, h_{k}}^{*}, K\right)(j=i, \cdots, i+q-1)$ on each element $K \in \mathcal{T}_{h_{k}}$ according to (4.1).

4. Construct $\mathcal{M}_{h_{k}} \subset \mathcal{T}_{h_{k}}$ by Algorithm 4.1. Then refine $\mathcal{M}_{h_{k}}$ to get a new conforming mesh $\mathcal{T}_{h_{k+1}}$ and construct finite element space $V_{h_{k+1}}$.

5. If $k<n$, do:

Obtain new eigenpair approximations $\left\{\lambda_{j, h_{k+1}}, u_{j, h_{k+1}}, u_{j, h_{k+1}}^{*}\right\}_{i=1}^{i+q-1} \in \mathbb{C} \times V_{h_{k+1}} \times V_{h_{k+1}}$ by Algorithm 3.3 on $\mathcal{T}_{h_{k+1}}$ :

- Set $\left\{\lambda_{j, h_{k}}^{(0)}, u_{j, h_{k}}^{(0)}, u_{j, h_{k}}^{*(0)}\right\}_{j=i}^{i+q-1}=\left\{\lambda_{j, h_{k-1}}, u_{j, h_{k-1}}, u_{j, h_{k-1}}^{*}\right\}_{j=i}^{i+q-1}$.

- Perform the following multigrid iterations for $\ell=0, \cdots, p-1$

$$
\left\{\lambda_{j, h_{k}}^{(\ell+1)}, u_{j, h_{k}}^{(\ell+1)}, u_{j, h_{k}}^{*(\ell+1)}\right\}_{j=i}^{i+q-1}=\text { EigenMG_Multi }\left(V_{H},\left\{\lambda_{j, h_{k}}^{(\ell)}, u_{j, h_{k}}^{(\ell)}, u_{j, h_{k}}^{*(\ell)}\right\}_{j=i}^{i+q-1}, V_{h_{k}}, m\right) .
$$

- Set $\left\{\lambda_{j, h_{k}}, u_{j, h_{k}}, u_{j, h_{k}}^{*}\right\}_{j=i}^{i+q-1}=\left\{\lambda_{j, h_{k}}^{(p)}, u_{j, h_{k}}^{(p)}, u_{j, h_{k}}^{*(p)}\right\}_{j=i}^{i+q-1}$.

- Let $k=k+1$ and go to step 3 .

Else, do:

Obtain new eigenpair approximations $\left\{\lambda_{j, h_{n}}^{(p)}, u_{j, h_{n}}^{(p)}, u_{j, h_{n}}^{*(p)}\right\}_{i=1}^{i+q-1} \in \mathbb{C} \times V_{h_{k+1}} \times V_{h_{k+1}}$ by Algorithm 3.3 on $\mathcal{T}_{h_{k+1}}$ :

- Set $\left\{\lambda_{j, h_{k}}^{(0)}, u_{j, h_{k}}^{(0)}, u_{j, h_{k}}^{*(0)}\right\}_{j=i}^{i+q-1}=\left\{\lambda_{j, h_{k-1}}, u_{j, h_{k-1}}, u_{j, h_{k-1}}^{*}\right\}_{j=i}^{i+q-1}$.

- Perform the following multigrid iterations for $\ell=0, \cdots, p-1$

$$
\left\{\lambda_{j, h_{k}}^{(\ell+1)}, u_{j, h_{k}}^{(\ell+1)}, u_{j, h_{k}}^{*(\ell+1)}\right\}_{j=i}^{i+q-1}=\text { EigenMG_Multi }\left(V_{H},\left\{\lambda_{j, h_{k}}^{(\ell)}, u_{j, h_{k}}^{(\ell)}, u_{j, h_{k}}^{*(\ell)}\right\}_{j=i}^{i+q-1}, V_{h_{k}}, m\right) .
$$

- Set $\left\{u_{j, h_{k}}, u_{j, h_{k}}^{*}\right\}_{j=i}^{i+q-1}=\left\{u_{j, h_{k}}^{(p)}, u_{j, h_{k}}^{*(p)}\right\}_{j=i}^{i+q-1}$.

- Compute the generalized Rayleigh quotient

$$
\lambda_{j, h_{n}}=\frac{a\left(u_{j, h_{n}}, u_{j, h_{n}}^{*}\right)}{b\left(u_{j, h_{n}}, u_{j, h_{n}}^{*}\right)} \quad(j=i, \cdots, i+q-1) .
$$

- Stop.

Finally, we get the eigenpair approximation

$$
\left\{\lambda_{j, h_{n}}, u_{j, h_{n}}, u_{j, h_{n}}^{*}\right\}_{j=i}^{i+q-1} \in \mathbb{C} \times V_{h_{n}} \times V_{h_{n}} .
$$




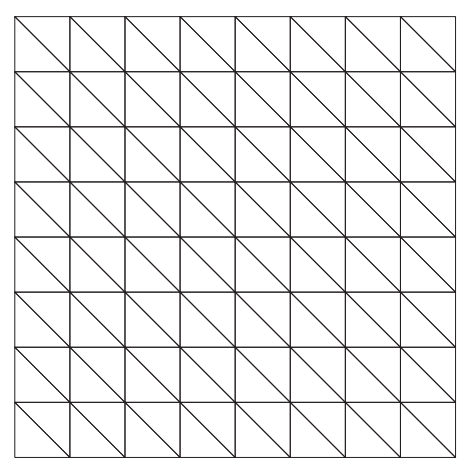

Figure 1. The initial/coarsest mesh for Example 1.

Remark 4.1. For the Algorithm 4.2, we can also obtain the related error reduction property and the optimal complexity analysis base on the asymptotic exactness of error estimators (cf. [45, Thm. 5.1 and 5.2]). Due to the simplicity of this paper, we omit these parts. More details, please refer to [23].

Corollary 4.2. Assume the conditions of Theorem 3.8 hold, then the work involved in Algorithm 4.2 has the following estimate

$$
\text { Total work } \leq \mathcal{O}\left(N_{n}+M_{H} \log N_{n}+M_{h_{1}}\right) .
$$

Futhermore, the complexity will be $\mathcal{O}\left(N_{n}\right)$ provided $\mathcal{O}\left(M_{H}\right) \ll \mathcal{O}\left(N_{n}\right)$ and $\mathcal{O}\left(M_{h_{1}}\right) \leq \mathcal{O}\left(N_{n}\right)$.

\section{Numerical RESUlts}

In this section, some numerical examples are presented to illustrate the efficiency of the full multigrid method proposed in Algorithm 3.2 and AFEM based on full multigrid adaptive finite element method 4.2 for nonselfadjoint Steklov eigenvalue problems, respectively. When $n(x)$ is a real function, $(2.2)$ is a selfadjoint eigenvalue problem. And, we choose $n(x)=4+4 \mathrm{i}$ in the following examples. In each level of the full multigrid scheme defined in Algorithm 3.2, 3.4 and 4.2, the parameters are set to be $m=3$ and $p=1$, respectively. In addition, we take 3 conjugate gradient smooth steps for the presmoothing and postsmoothing iteration step in the multigrid iteration in Step 1 of Algorithm 3.1 and 3.3.

\subsection{Non-selfadjoint Steklov eigenvalue problem on square domain}

We first consider non-selfadjoint Steklov eigenvalue problem (2.1) with $\mathcal{A}(x)=1$ and $n(x)=4+4$ i defined on square domain $\Omega=\left(-\frac{\sqrt{2}}{2}, \frac{\sqrt{2}}{2}\right)^{2}$. Hence $\sigma=1$. The sequence of linear finite element spaces are constructed on the series of meshes which are produced by the regular refinement with $\beta=2$ (producing $\beta^{2}$ subelements). In this example, we choose a mesh which is generated by uniform refinement as the initial mesh $\mathcal{T}_{h_{1}}$ and the coarsest mesh $\mathcal{T}_{H}$ to produce a sequence of finite element spaces for investigating the convergence behaviors. Figure 1 shows this initial meshes $\left(h_{1}=H=\sqrt{2} / 8\right)$

Since the exact eigenvalue is unknown, we use the accurate enough approximation $[0.686553+$ $2.495294 \mathrm{i},-0.343047+0.850747 \mathrm{i},-0.343047+0.850747 \mathrm{i},-0.950110+0.540097 \mathrm{i}]$ given by the extrapolation method (see, e.g. [28]) as the first four exact eigenvalues (sorted by real part) to investigate the errors. Algorithm 3.2 is applied to solve (2.1).

Figure 2 gives the corresponding numerical results for the first eigenvalue $\lambda_{1}=0.686553+2.495294$ i. From Figure 2, we find that the full multigrid can obtain the optimal error estimates as the expected one for the direct finite element method, which confirms with the convergence Theorem 3.6 for Algorithm 3.2. 

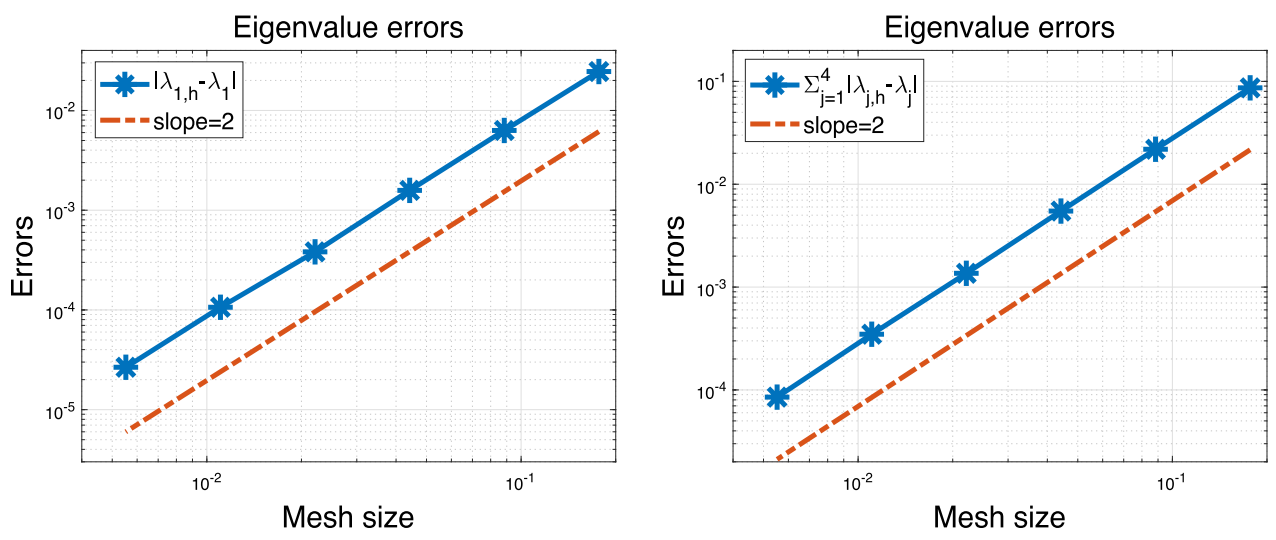

Figure 2. The errors of the full multigrid method for the first eigenvalue $\lambda_{1}$ (left) and first four eigenvalues $\lambda_{1}, \cdots, \lambda_{4}$ (right) on the square domain for the initial mesh in Figure 1.

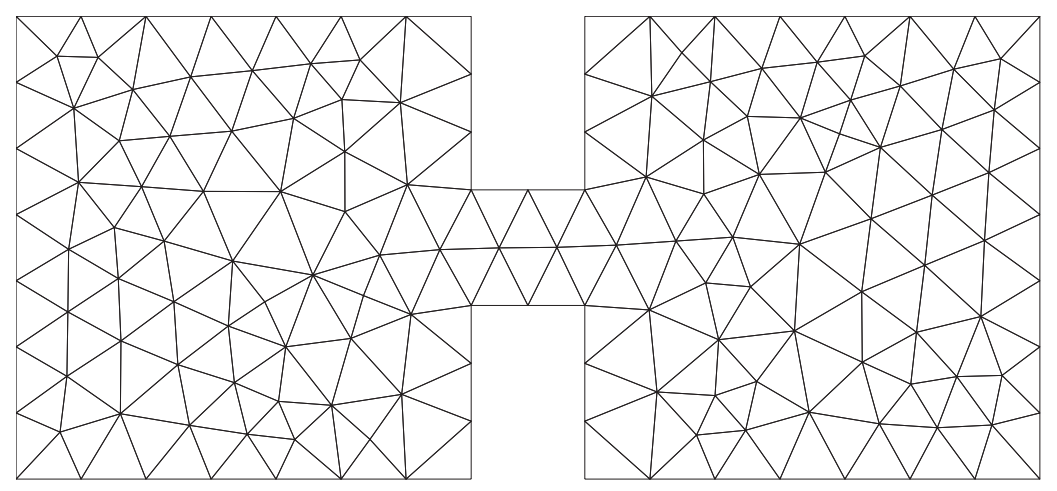

Figure 3. The initial/coarsest mesh for Example 2.

We also check the convergence behavior for multiple eigenvalue approximations with Algorithm 3.4. Here the first four eigenvalues are investigated. Similarly, we use the same initial mesh shown in Figure 1. The corresponding numerical results are given in Figure 2, which also exhibits the optimal convergence rate of the full multigrid scheme Algorithm 3.4.

\subsection{Non-selfadjoint Steklov eigenvalue problem on dumbbell shaped domain}

In order to show our full multigrid method can work well with adaptive mesh (Algorithm 4.2), we discuss the non-selfadjoint Steklov eigenvalue problem with $\mathcal{A}(x)=1$ and $n(x)=4+4 \mathrm{i}$ defined on a dumbbell shaped domain $\Omega=(0, \pi)^{2} \cup\left[\pi, \frac{5}{4} \pi\right] \times\left(\frac{3}{8} \pi, \frac{5}{8} \pi\right) \cup\left(\frac{5}{4} \pi, \frac{9}{4} \pi\right) \times(0, \pi)$. The initial/coarsest mesh for this dumbbell shaped domain is given in Figure 3 which is generated by Delaunay method to produce a sequence of finite element spaces for investigating the convergence behaviors.

It is easy to know that reentrant corners of the dumbbell domain result in the singularities of the eigenfunctions. The convergence order for eigenfunction approximations is less than 1 by the linear finite element method, which is the order predicted by the theory for regular eigenfunctions $(\sigma<1)$. We consider to use the adaptive Algorithm 4.2 to solve this problem. Figure 4 shows the mesh after 15 adaptive refinements.

Since the exact solution is unknown, we use the accurate enough approximation $[-0.245575-$ $1.723065 \mathrm{i},-0.246998-1.720376 \mathrm{i},-0.492629-1.284891 \mathrm{i},-0.570899-1.344935 \mathrm{i},-0.623435-1.198207 \mathrm{i}]$ given 


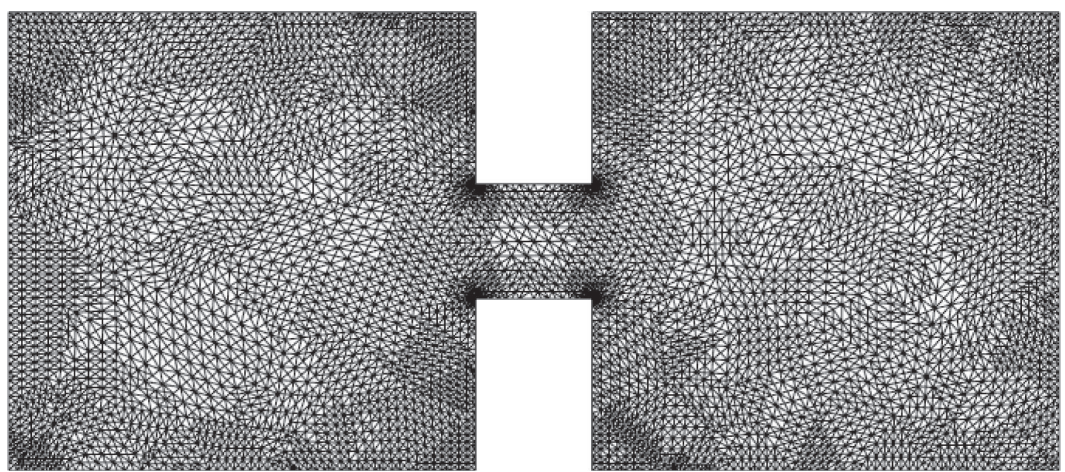

FIGURE 4 . The mesh after 15 adaptive refinements.
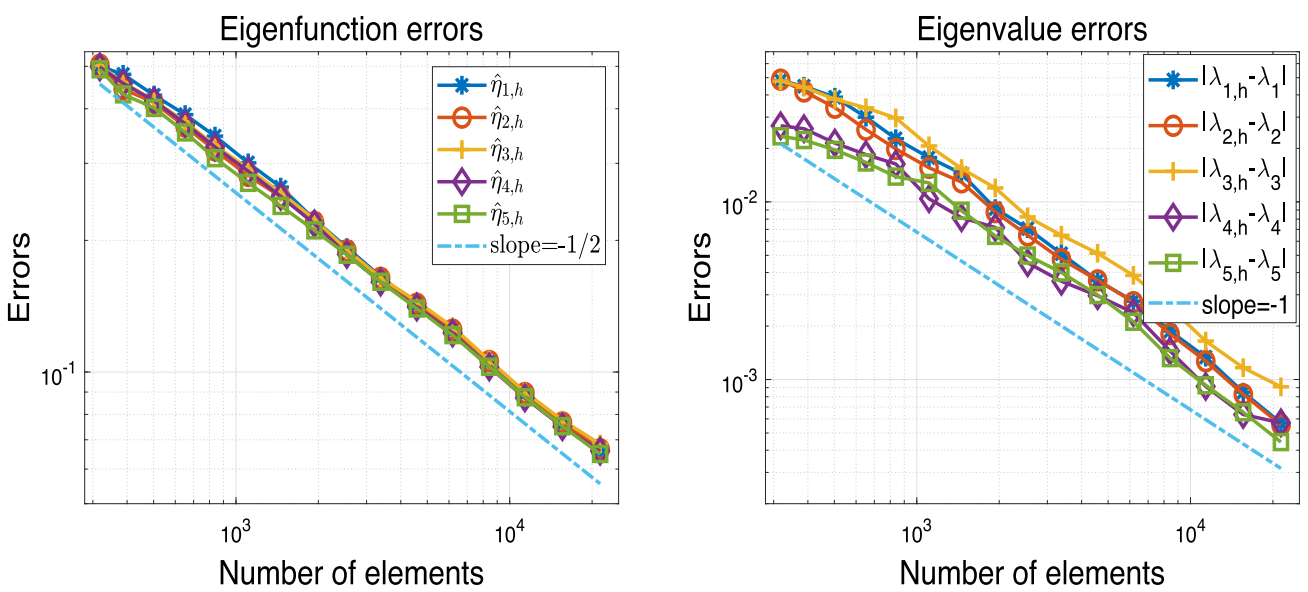

FiguRE 5. The errors of the adaptive full multigrid algorithm for the first five eigenfunction approximations (left) and eigenvalue approximations (right) where $\hat{\eta}_{j, h}(j=1, \cdots, 5)$ denote the $j$-th posterior error estimator $\hat{\eta}_{h}\left(u_{j, h}, u_{j, h}^{*}, \mathcal{T}_{h}\right)$ and $\lambda_{j, h}(j=1, \cdots, 5)$ denote the current approximation of the $j$-th eigenvalue $\lambda_{j}$.

by the extrapolation method (see, e.g. [28]) as the first five exact eigenvalues to investigate the errors. First, we investigate the convergent rate of the adaptive posterior error estimator $\hat{\eta}_{h}\left(u_{h}, u_{h}^{*}, \omega\right)\left(\omega \subset \mathcal{T}_{h}\right)$ defined in (4.2). Figure 5 presents the corresponding numerical results for the first five eigenfunction approximations. Here, we use $\hat{\eta}_{j, h}$ to denote the $j$-th error estimator $\hat{\eta}_{h}\left(u_{j, h}, u_{j, h}^{*}, \mathcal{T}_{h}\right)$. The error estimate of eigenvalues are given in Figure 5, which shows that our multilevel iteration method combines well with the adaptive finite element method naturally and Algorithm 4.2 has the optimal convergence rate.

\subsection{Non-selfadjoint Steklov eigenvalue problem with discontinuous coefficient}

In this example, we consider the non-selfadjoint Steklov eigenvalue problem with with discontinuous coefficient. Define computational domain $\Omega=\left(-\frac{\sqrt{2}}{2}, \frac{\sqrt{2}}{2}\right)^{2}$, discontinuous coefficient

$$
\mathcal{A}(x)= \begin{cases}100, & \text { in the } 1 \text { st and 3rd quadrants, } \\ 1, & \text { in the } 2 \text { nd and 4th quadrants }\end{cases}
$$

and $n(x)=4+4 \mathrm{i}$. The initial/coarsest mesh for this square domain is the same as the one in Example 5.1. 


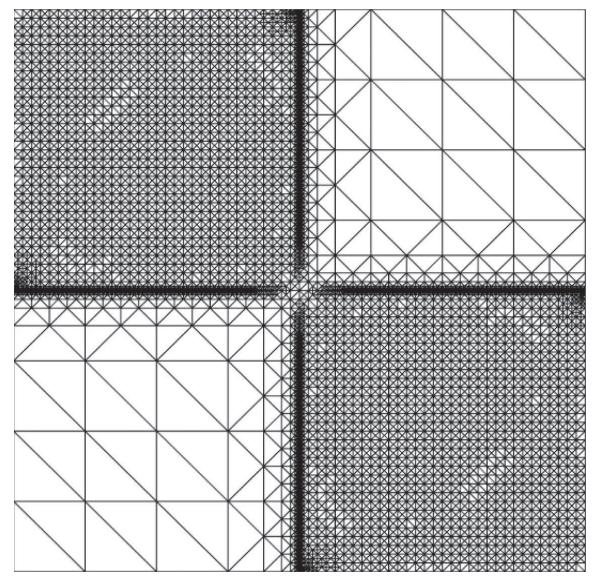

Figure 6. The mesh after 17 adaptive refinements.
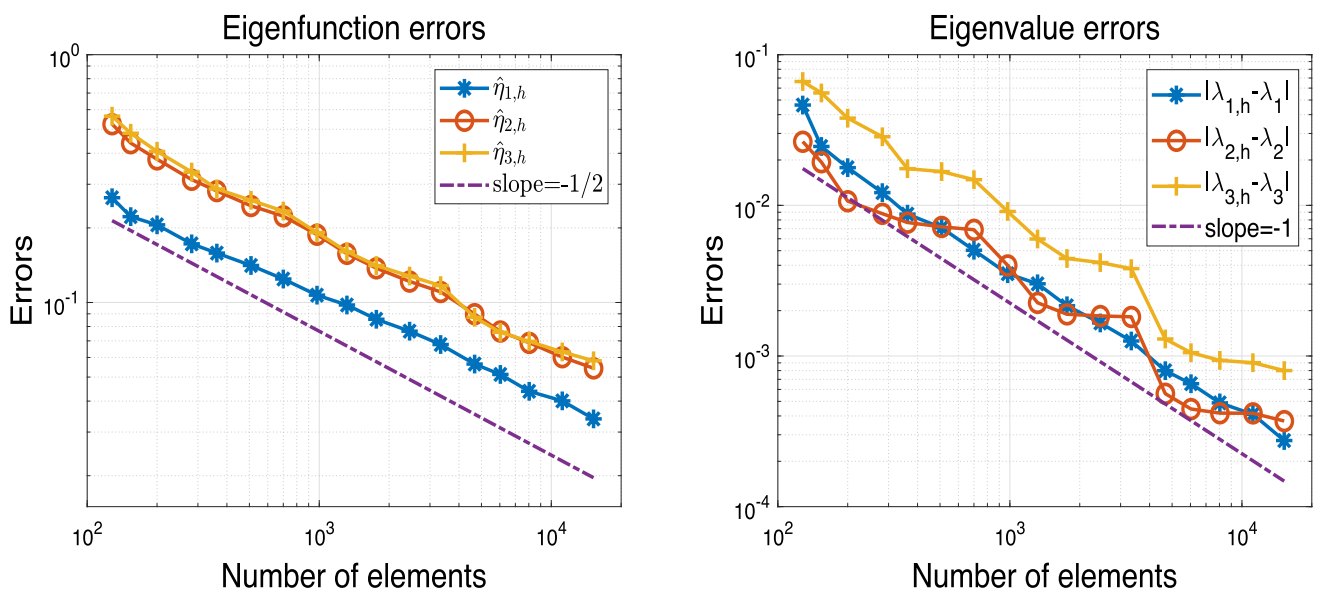

FigURE 7. The errors of the adaptive full multigrid algorithm for the first three eigenfunction approximations (left) and eigenvalue approximations $($ right $)$ where $\hat{\eta}_{j, h}(j=1, \cdots, 3)$ denote the $j$-th posterior error estimator $\hat{\eta}_{h}\left(u_{j, h}, u_{j, h}^{*}, \mathcal{T}_{h}\right)$ and $\lambda_{j, h}(j=1, \cdots, 3)$ denote the current approximation of the $j$-th eigenvalue $\lambda_{j}$.

It is easy to know that the singularities is on the interface. The convergence order for eigenfunction approximations is less than $1(\sigma<1)$ by the linear finite element method, which is the order predicted by the theory for regular eigenfunctions. We also consider to use the adaptive Algorithm 4.2 to solve this problem. Figure 6 shows the mesh after 17 adaptive refinements.

Since the exact solution is unknown, we use the accurate enough approximation $[1.451305-$ $1.741234 \mathrm{i},-0.942417-0.542984 \mathrm{i},-1.563818-0.563167 \mathrm{i}]$ given by the extrapolation method (see, e.g. [28]) as the first three exact eigenvalues to investigate the errors.

Figure 7 shows the corresponding numerical results by Algorithm 4.2. From Figure 7, we can find that Algorithm 4.2 is able to obtain the optimal error estimate. It shows that Algorithm 4.2 is efficient for solving non-selfadjoint Steklov eigenvalue problems with discontinuous coefficient. 


\section{Concluding Remarks}

In this paper, a type of full multigrid method is designed to solve non-selfadjoint eigenvalue problems based on the multigrid for boundary value problems and the multilevel correction scheme for eigenvalue problems. Furthermore, when the number of smoothing steps is chosen appropriately, our method can reach the optimal convergence rate with the almost optimal computing complexity. At last, we propose a new type of AFEM for multiple eigenvalues based on full multigrid with the almost optimal computing complexity. Three numerical experiments validate the optimality and show that the proposed algorithms can also compute multiple eigenvalues and solve the eigenvalue problems with complex vector.

Acknowledgements. The authors thank Prof. Hehu Xie for fruitful cooperations on eigenvalue computations that have motivated this work and thank the anonymous referees for their helpful comments and suggestions on this work. This work was supported in part by the National Natural Science Foundation of China (Grant No. 11801021, 12001402).

\section{REFERENCES}

[1] M. Armentano and C. Padra, A posteriori error estimates for the Steklov eigenvalue problem. Appl. Numer. Math. 58 (2008) 593-601.

[2] I. Babuška and J. Osborn, Eigenvalue Problems, edited by J. Lions and P. Ciarlet, Vol. II. In: Handbook of numerical analysis, Finite element methods (Part 1). North-Holland, Amsterdam (1991) 641-787.

[3] R.E. Bank and T. Dupont, An optimal order process for solving finite element equations. Math. Comput. 36 (1981) 35-51.

[4] H. Bi and Y. Yang, A two-grid method of the non-conforming Crouzeix-Raviart element for the Steklov eigenvalue problem. Appl. Math. Comput. 217 (2011) 9669-9678.

[5] H. Bi, Y. Zhang and Y. Yang, Two-grid discretizations and a local finite element scheme for a non-selfadjoint Stekloff eigenvalue problem. Comput. Math. Appl. 79 (2020) 1895-1913.

[6] J. Bramble and J. Osborn, Approximation of Steklov Eigenvalues of Non-selfadjoint Second Order Elliptic Operators, edited by A. Aziz, In: Mathematical foundations of the finite element method with applications to PDEs. Academic Press, New York (1972) 387-408.

[7] J. Bramble and J. Pasciak, New convergence estimates for multigrid algorithms. Math. Comput. 49 (1987) 311-329.

[8] S. Brenner and L. Scott, The Mathematical Theory of Finite Element Methods. Springer-Verlag, New York (1994).

[9] F. Cakoni, D. Colton, S. Meng and P. Monk, Steklov eigenvalues in inverse scattering. SIAM J. Appl. Math. 76 (2016) $1737-1763$.

[10] J.A. Canavati and A.A. Minzoni, A discontinuous Steklov problem with an application to water waves. J. Math. Anal. Appl. 69 (1979) 540-558.

[11] L. Cao, L. Zhang, W. Allegretto and Y. Lin, Multiscale asymptotic method for Steklov eigenvalue equations in composite media. SIAM J. Numer. Anal. 51 (2013) 273-296.

[12] C. Carstensen and J. Gedicke, An adaptive finite element eigenvalue solver of asymptotic quasi-Optimal computational complexity. SIAM J. Numer. Anal. 50 (2012) 1029-1057.

[13] J.M. Cascon, C. Kreuzer, R.H. Nochetto and K.G. Siebert, Quasi-optimal convergence rate for an adaptive finite element method. SIAM J. Numer. Anal. 46 (2008) 2524-2550.

[14] Z. Chen and S. Dai, On the efficiency of adaptive finite element methods for elliptic problems with discontinous coefficients. SIAM J. Sci. Comput. 24 (2002) 443-462.

[15] H. Chen, H. Xie and F. Xu, A full multigrid method for eigenvalue problems. J. Comput. Phys. 322 (2016) $747-759$.

[16] P. Ciarlet, The Finite Element Method for Elliptic Problems. North-Holland, Amsterdam (1978).

[17] K. Cliffe, E. Hall and P. Houston, Adaptive discontinuous Galerkin methods for eigenvalue problems arising in incompressible fluid flows, SIAM J. Sci. Comput. 31 (2008) 4607-4632.

[18] X. Dai, J. Xu and A. Zhou, Convergence and optimal complexity of adaptive finite element eigenvalue computations. Numer. Math. 110 (2008) 313-355.

[19] S. Giani and I.G. Graham, A convergent adaptive method for elliptic eigenvalue problems. SIAM J. Numer. Anal. 47 (2009) $1067-1091$.

[20] W. Hackbusch, Multi-Grid Methods and Applications. Vol.4 of: Computational Mathematics. Springer-Verlag, BerlinHeidelberg (1985).

[21] X. Han, H. Xie and F. Xu, A cascadic multigrid method for eigenvalue problem. J. Comput. Math. 35 (2017) 56-72.

[22] V. Heuveline and R. Rannacher, A posteriori error control for finite element approximations of elliptic eigenvalue problems. Adv. Comput. Math. 15 (2001) 107-138.

[23] Q. Hong, H. Xie and F. Xu, A Multilevel correction type of adaptive finite element method for eigenvalue problems. SIAM J. Sci. Comput. 40 (2018) A4208-A4235.

[24] G. Hu, H. Xie and F. Xu, A multilevel correction adaptive finite element method for Kohn-Sham equation. J. Comput. Phys. 355 (2018) 436-449. 
[25] X. Ji, J. Sun and H. Xie, A multigrid method for Helmholtz transmission eigenvalue problems. J. Sci. Comput. 60 (2014) $276-294$.

[26] S. Jia, H. Xie, M. Xie and F. Xu, A full multigrid method for nonlinear eigenvalue problems. Sci. China Math. 59 (2016) $2037-2048$

[27] N. Kuznetsov, T. Kulczycki, M. Kwaśnicki, A. Nazarov, S. Poborchi, I. Polterovich and B. Siudeja, The legacy of Vladimir Andreevich Steklov. Not. Am. Math. Soc. 61 (2014) 9-22.

[28] Q. Lin and J. Lin, Finite Element Methods: Accuracy and Inprovement. Science Press, Beijing (2006).

[29] Q. Lin and H. Xie, A multi-level correction scheme for eigenvalue problems. Math. Comput. 84 (2015) 71-88.

[30] J. Liu, Y. Liu and J. Sun, An inverse medium problem using Stekloff eigenvalues and a Bayesian approach. Inverse Prob. 35 (2019) 094004.

[31] J. Liu, J. Sun and T. Turner, Spectral indicator method for a non-selfadjoint Steklov eigenvalue problem. J. Sci. Comput. 79 (2019) 1814-1831.

[32] J. Meng and L. Mei, Discontinuous Galerkin methods of the non-selfadjoint Steklov eigenvalue problem in inverse scattering. Appl. Math. Comput. 381 (2020) 125-307.

[33] P. Morin, R.H. Nochetto and K. Siebert, Convergence of adaptive finite element methods. SIAM Rev. 44 (2002) 631-658.

[34] A.D. Russo and A.E. Alonso, A posteriori error estimates for nonconforming approximations of Steklov eigenvalue problem. Comput. Appl. Math. 62 (2011) 4100-4117.

[35] R. Verfürth, A Review of a Posteriori Error Estimation Andadaptive Mesh-refinement Techniques. Wiley-Teubner, New York (1996).

[36] H. Xie, A multigrid method for eigenvalue problem. J. Comput. Phys. 274 (2014) 550-561.

[37] H. Xie, A type of multilevel method for the Steklov eigenvalue problem. IMA J. Numer. Anal. 34 (2014) $592-608$.

[38] H. Xie and X. Wu, A multilevel correction method for interior transmission eigenvalue problem. J. Sci. Comput. 72 (2017) $586-604$.

[39] H. Xie and M. Xie, A multigrid method for the ground state solution of Bose-Einstein condensates. Commun. Comput. Phys. 19 (2016) 648-662.

[40] H. Xie and M. Xie, Computable error estimates for ground state solution of Bose-Einstein condensates. J. Sci. Comput. 81 (2019) 1072-1087.

[41] H. Xie, M. Xie, X. Yin and M. Yue, Computable error estimates for a nonsymmetric eigenvalue problem. East Asian J. Appl. Math. 7 (2017) 583-602.

[42] H. Xie and Z. Zhang, A multilevel correction scheme for nonsymmetric eigenvalue problems by finite element methods. arXiv:1505.06288 (2015) http://arxiv.org/abs/1505.06288.

[43] H. Xie and T. Zhou, A multilevel finite element method for Fredholm integral eigenvalue problems. J. Comput. Phys. 303 (2015) 173-184.

[44] F. Xu, H. Xie and N. Zhang, A parallel augmented subspace method for eigenvalue problems. SIAM J. Sci. Comput. 42 (2020) A2655-A2677.

[45] J. Xu and Z. Zhang, Analysis of recovery type a posteriori error estimators for mildly structured grids. Math. Comput. 73 (2003) 1139-1152.

[46] F. Xu, M. Yue, Q. Huang and H. Ma, An asymptotically exact a posteriori error estimator for non-selfadjoint Steklov eigenvalue problem. Appl. Numer. Math. 156 (2020) 210-227.

[47] Y. Yang, J. Han and H. Bi, Error estimates and a two grid scheme for approximating transmission eigenvalues. arXiv:1506.06486v2 (2016) http://arxiv.org/abs/1506.06486v2.

[48] M. Yue, H. Xie and M. Xie, A cascadic multigrid method for nonsymmetric eigenvalue problem. Appl. Numer. Math. 146 (2019) 55-72.

[49] Y. Zeng and F. Wang, A posteriori error estimates for a discontinuous Galerkin approximation of Steklov eigenvalue problems. Appl. Math. 62 (2017) 243-267.

[50] S. Zhang, Y. Xi and X. Ji, A multi-level mixed element method for the eigenvalue problem of biharmonic equation. J. Sci. Comput. 75 (2018) 1415-1444.

[51] Y. Zhang, H. Bi and Y. Yang, A multigrid correction scheme for a new Steklov eigenvalue problem in inverse scattering. Int. J. Comput. Math. (2019) DOI:10.1080/00207160.2019.1622686.

[52] Z. Zhang and A. Naga, A new finite element gradient recovery method: superconvergence property. SIAM J. Sci. Comput. 26 (2005) 1192-1213.

[53] O.C. Zienkiewicz and J. Zhu, The superconvergent patch recovery and a posteriori error esti- mates. Part 1: The recovery technique. Int. J. Numer. Methods Eng. 33 (1992) 1331-1364. 


\section{Subscribe to Open (S2O) \\ A fair and sustainable open access model}

This journal is currently published in open access under a Subscribe-to-Open model (S2O). S2O is a transformative model that aims to move subscription journals to open access. Open access is the free, immediate, online availability of research articles combined with the rights to use these articles fully in the digital environment. We are thankful to our subscribers and sponsors for making it possible to publish this journal in open access, free of charge for authors.

\section{Please help to maintain this journal in open access!}

Check that your library subscribes to the journal, or make a personal donation to the $\mathrm{S} 2 \mathrm{O}$ programme, by contacting subscribers@edpsciences.org

More information, including a list of sponsors and a financial transparency report, available at: https://www. edpsciences.org/en/maths-s2o-programme 